Portland State University

PDXScholar

Fall 12-10-2013

\title{
Application of Support Vector Machine in Predicting the Market's Monthly Trend Direction
}

\author{
Ali Alali \\ Portland State University
}

Follow this and additional works at: https://pdxscholar.library.pdx.edu/open_access_etds

Part of the Electrical and Computer Engineering Commons Let us know how access to this document benefits you.

Recommended Citation

Alali, Ali, "Application of Support Vector Machine in Predicting the Market's Monthly Trend Direction" (2013). Dissertations and Theses. Paper 1496.

https://doi.org/10.15760/etd.1495

This Thesis is brought to you for free and open access. It has been accepted for inclusion in Dissertations and Theses by an authorized administrator of PDXScholar. Please contact us if we can make this document more accessible: pdxscholar@pdx.edu. 
Application of Support Vector Machine in Predicting the Market's Monthly Trend Direction

by

Ali Alali

A thesis submitted in partial fulfillment of the requirements for the degree of

\author{
Master of Science \\ in \\ Electrical and Computer Engineering
}

Thesis Committee:

Richard Tymerski, Chair

$\mathrm{Fu} \mathrm{Li}$

Xiaoyu Song

Portland State University

2013 
(C) 2013 Ali Alali 


\begin{abstract}
In this work, we investigate different techniques to predict the monthly trend direction of the S\&P 500 market index. The techniques use a machine learning classifier with technical and macroeconomic indicators as input features. The Support Vector Machine (SVM) classifier was explored in-depth in order to optimize the performance using four different kernels; Linear, Radial Basis Function (RBF), Polynomial, and Quadratic. A result found was the performance of the classifier can be optimized by reducing the number of macroeconomic features needed by $30 \%$ using Sequential Feature Selection. Further performance enhancement was achieved by optimizing the RBF kernel and SVM parameters through gridsearch. This resulted in final classification accuracy rates of $62 \%$ using technical features alone with gridsearch and $60.4 \%$ using macroeconomic features alone using Rankfeatures.
\end{abstract}




\section{Table of Contents}

Abstract

List of Tables

vi

List of Figures

ix

Chapter 1: Introduction

1.1 Problem Statement

1.2 Objective

1.3 Thesis Format

Chapter 2 Background Information and Literature Overview

2.1 Macroeconomic Data

2.1.1 Dividend Price Ratio

2.1.2 Dividend Yield

2.1.3 Earnings to Price Ratio 
2.1.13 Inflation 
4.1.6 Frequent Index's Price Oscillation 26

4.2 Classifier Data Processing Kernel 26

$\begin{array}{ll}\text { Chapter 5: Experiments and Results } & 27\end{array}$

$\begin{array}{ll}\text { 5.1 SVM Classification } & 27\end{array}$

$\begin{array}{ll}\text { 5.1.1 Macroeconomic Features } & 27\end{array}$

5.1.2 Technical Features $\quad 36$

5.2 SVM RBF Kernel Parameters Selection and Optimization 44

5.3 Feature Selection and Dimensionality Reduction 46

$\begin{array}{ll}\text { 5.3.1 Sequential Feature Selection } & 47\end{array}$

5.3.1.1 Sequential Feature Selection with Macroeconomic Features $\quad 47$

5.3.1.2 Sequential Feature Selection with Technical Features 49 
5.6 Comparison between Predictions Based on Basic Assumptions and SVM

6.1 Conclusion 
List of Tables

Table 2.1 Example for normalizing simple data with zscore

Table 2.2 Example for normalizing simple data with normc

Table 2.3 Example for normalizing simple data with normalize

Table 4.1 Dates of mismatched earnings

Table 5.1 Results for SVM classifier using Macroeconomic data and zscore normalization

Table 5.2 Results for SVM classifier using Macroeconomic data and normc normalization

Table 5.3 Results for SVM classifier using Macroeconomic data with 'normalize' function

Table 5.4 Macroeconomic Features Out-of-sample SVM predictions vs. actual realization

Table 5.5 Results for SVM classifier using technical features and zscore normalization

Table 5.6 Results for SVM classifier using technical features and normc normalization

Table 5.7 Results for SVM classifier using technical features and normalize function

Table 5.8 Technical Features Out-of-sample SVM predictions vs. actual realization 
Table 5.9 Macroeconomic Features Significance Ranking using Sequential Feature and linear kernel

Table 5.10 Sequential Feature Selection for macroeconomic data with Linear Kernel

Table 5.11 Macroeconomic Features Significance Ranking using Sequential

Feature and RBF kernel

Table 5.12 Sequential Feature Selection accuracy for macroeconomic data and 'zscore normalization and RBF Kernel

Table 5.13 Sequential Feature Selection for technical data and linear kernel

Table 5.14 Sequential Feature Selection for technical data and 'zscore' normalization and Linear Kernel

Table 5.15 Sequential Feature Selection for technical data and RBF kernel

Table 5.16 Sequential Feature Selection for technical data and 'zscore' normalization and RBF Kernel

Table 5.17 Macroeconomic features significance with Rankfeatures

Table 5.18 Rankfeatures Accuracy with macroeconomic features

Table 5.19 Technical features significance with Rankfeatures 
Table 5.22 Summary of the performance results using macroeconomic features

Table 5.24 Results for comparing the classification accuracy during the full out-of-sample period

Table 5.25 Results for comparing the classification accuracy during the first economic crisis period

Table 5.26 Results for comparing the classification accuracy during the last economic crisis period 
List of Figures

Figure 4.1 S\&P 500 Monthly index over the training and testing period 22

Figure 4.2 S\&P 500 DE movements over the training and testing period 22

Figure 4.3 S\&P 500 EP movements over the training and testing period 23

Figure 4.4 S\&P 500 INFL movements over the training and testing period 23

Figure 5.1 In-sample macroeconomic error rates vs. parameters change 45

Figure 5.2 In-sample technical error rates vs. parameters change $\quad 46$

Figure 5.3 S\&P 500 Index price over the first economic crisis

(October 2000 - September 2002)

60

Figure 5.4 S\&P 500 Index price during the last economic crisis

(October 2007 - July 2009)

61 
Chapter 1 Introduction

Appearing in The New Palgrave: A dictionary of Economics, it was described that "The Efficient Markets Hypothesis $(E M H)$ maintains that market prices fully reflect all available information. Developed independently by Paul A. Samuelson and Eugene F. Fama in the 1960s, this idea has been applied extensively to theoretical models and empirical studies of financial securities prices, generating considerable controversy as well as fundamental insights into the price-discovery process. The most enduring critique comes from psychologists and behavioral economists who argue that the EMH is based on counterfactual assumptions regarding human behavior, that is, rationality. Recent advances in evolutionary psychology and the cognitive neurosciences may be able to reconcile the EMH with behavioral anomalies [1]." Another financial theory is called the Random Walk Hypothesis $(\mathrm{RWH})$ which supports the $\mathrm{EMH}$. The RWH proposes the market prices change randomly which results in it being unpredictable.

Recently, studies show that the ability to predict market movement based on macroeconomic and technical analysis is possible. Macroeconomic analysis measures the health of a certain company and decides the value for a given business in order to predict if in the future the price will change in a certain direction. Technical analysis on the other hand uses the markets' historical prices and volume in order to create an interpretation of the market's state. With use of technical analysis, an analyst can convert the prices into various 
indicators in order to understand the market state better and possibly make better prediction decisions.

The purpose of this work was to explore the techniques used to predict the market's monthly trend direction using macroeconomic and technical analysis. Using this data, an in-depth investigation using machine learning techniques was performed in order to create a model for predicting the market's movement. The result of this thesis shows that macroeconomic and technical information can be used as input to a machine learning classifier to create a prediction model that predicts if the market's movement for the following month is 'up' or 'down'.

\subsection{Problem Statement}

Predicting the monthly direction of the market is a problem faced by many investors. The work presented in this thesis develops a prediction model that can be used to help trading securities safer and cause less risk involved when making an investing decision.

\subsection{Objective}

The objective of this work was to develop a market prediction model that can successfully predict the monthly returns on a market to gain profit and reduce the risk involved. This was achieved by constructing a market prediction simulator with exploration of many different techniques to optimize the model's 
performance. This model was evaluated by noting the monthly in-sample and out-of-sample classification accuracy.

\subsection{Thesis Format}

The following includes a literature of background information and related techniques of market data classification (Chapter 2); a description of goals, hypothesis and evaluation methods (Chapter 3); an explanation of design (Chapter 4); and a thorough description of all experimental prediction strategies with results (Chapter 5). 
Chapter 2 Background Information and Literature Overview

The potential use of different data types and systems to predict the market's trend direction has increased in the recent years with many different techniques available. This thesis provides an explanation and overview of macroeconomic and technical data used with machine learning techniques to predict the direction of the market's monthly trend.

\subsection{Macroeconomic Data}

Macroeconomic data are measurements and indicators used to describe the current or previous economy's state of a country [2]. The macroeconomic data measures the overall health of an economy. Ability to obtain the macroeconomic data is not as simple as obtaining technical data, the other source of data used to analyze the state of the market and economy.

Macroeconomic Indicators [3]:

\subsubsection{Dividend Price Ratio}

The dividend per share paid to the share on a stock exchange paid previously, used as a measure of the potential investment of a certain stock.

\subsubsection{Dividend Yield}

It is a ratio of the dividends to the price per index that explains how much the pay out in dividends relative to the index price. 


\subsubsection{Earnings to Price Ratio}

Earnings are the amount of profit that a company produces in a specific period which shows the company's profitability. An Earnings to Price is the valuation of an index's earnings to its price.

\subsubsection{Stock Variance}

Stock Variance is a measure of volatility from an average which is used to measure the risk when purchasing a certain index.

\subsubsection{Book-to-Value}

Book-to-Value is a ratio of a company's historical cost to the company's market value which can be found through its market capitalization. It helps to identify if the index is overvalued or undervalued. A ratio above 1 indicates the index is undervalued while less than 1 is overvalued.

\subsubsection{Net Equity Expansion}

Net Equity Expansion is "the ratio of 12-month moving sums of net issues by NYSE listed stocks divided by the total end-of-year market capitalization of NYSE stocks."

\subsubsection{Treasury-bill}

Treasury bill is a short-dated government security that yields no interest but is offered at discounted prices on its redemption price. 


\subsubsection{Long Term Yield}

It is the percentage of return of investment on the debt responsibilities of the U.S. government.

\subsubsection{Long Term Return}

\subsubsection{Term Spread}

\subsubsection{Default Yield Spread}

Default yield spread is the difference between the quoted rates of return on two different investments. In our case, it is used between AAA and BAA-rated bonds.

\subsubsection{Default Return Spread}

\subsubsection{Inflation}

Inflation is the rate at which the general level of prices for goods and services increases and a fall in the purchasing power.

\subsection{Technical Data}

Technical analysis is the study of financial markets behavior. Technical analysis consists of evaluating historical prices in order to create technical indicators which indicate the current or past state of a certain security in market [4]. In this 
work, we use some of the many common technical indicators as input features to the classifier.

Technical Indicators used in this work:

\subsubsection{Relative Strength Index}

An indicator attempts to identify if it is an overbought or oversold market by comparing the magnitude of resent gains to losses [6]. It is calculated as the following:

$$
\begin{aligned}
& R S I=100-\left(\frac{100}{R S}\right) \\
& R S=\frac{\text { Average Gain }}{\text { Average LosS }}
\end{aligned}
$$

\subsubsection{Bollinger Bands}

Bollinger Bands are volatility bands based on standard deviation which are placed above and below a moving average. They are used to determine the strength of the trend [7]. They're calculated by the following formulas:

1. Middle Band $=\mathrm{t}$-day simple moving average $(\mathrm{SMA})$.

2. Upper Band $=\mathrm{t}$-day SMA $+(\mathrm{t}$-day standard deviation of price $\times 2)$

3. Lower Band = t-day SMA - (t-day standard deviation of price $\times 2$ ) 


\subsubsection{Stochastic}

It is an indicator to tell if the market is oversold or overbought by comparing the price of a certain security over a given period of time. This is done by:

$$
\begin{gathered}
\% K=100\left[\frac{\mathrm{C}(\mathrm{t})-\mathrm{L}(14)}{\mathrm{H}(14)-\mathrm{L}(14)}\right] \\
\% D=3-\text { period moving average of } \% \mathrm{~K}
\end{gathered}
$$

$\mathrm{C}=$ the most recent closing price.

$L(14)=$ the low of the 14 previous trading sessions.

$H(14)=$ the highest price traded during the same 14-day period.

\subsubsection{Simple Moving Average}

This indicator is formed by computing the average index price over a given period. In other words, it is defined as the $\mathrm{N}$ day's sum of closing price divided by $\mathrm{N}[9]$.

\subsubsection{Momentum}

It is an indicator that measures the change of a security's price over a given time period. It is defined by:

$$
\text { Momentum }=\frac{\operatorname{Price}(\mathrm{N})}{\operatorname{Price}(\mathrm{N}-\mathrm{t})} * 100
$$




\subsection{Classifier}

Classifiers are machine learning algorithms that can be used to classify a problem given a set of data. This work uses and investigates the Support Vector Machine (SVM) classifier closely to classify up or down periods given two different types of data sets as inputs; Macroeconomic and technical data.

\subsubsection{Support Vector Machine}

A Support Vector Machine is a supervised learning algorithm that can use given data to solve certain problems by attempting to convert them into linearly separable problems [11]. The SVM is given input data called training data sets that are linked to binary outputs in order to classify new observation to one of the two classes by creating a separating hyperplane [12]. Through the created hyperplane, the algorithm then labels new examples. In this work, we perform SVM training and classification using Matlab with functions 'svmtrain' and 'svmclassify'. Four different kernels are used in this work; Linear, Radial Basis Function (RBF), Polynomial, and Quadratic. These functions are provided in the

Statistics Toolbox as of the Matlab version 2013a. The mathematical formulation for each kernel is shown here [14]:

- Linear: $K(x, y)=w(x, y)+b$. The vector $w$ is known as the weight vector and $b$ is called the bias.

- Radial basis function - RBF: For some positive number $\sigma$ : 
o $K(x, y)=\exp \left[\frac{-|| x i-x j||^{2}}{2 \sigma^{2}}\right]$.

o $\quad x_{i}$ and $x_{j}$ will have either one becoming the support vector and the other will be the testing data point.

- Polynomial: For some positive integer $d$ :

o $K(x, y)=\left(1+\langle x \cdot y>)^{d}\right.$. Where $\mathrm{d}$ is the polynomial's degree

- Quadratic: $K(x, y)=(<x . y>)^{2}$.

\subsection{A Literature Overview}

This work performs a study on techniques used to predict the market's trend direction using macroeconomic and technical data and feeding this data to a machine learning classifier such as the SVM in our case.

\subsubsection{SVM Prediction System}

In the paper "Predicting S\&P 500 Returns Using Support Vector Machines: Theory and Empirics," the author mentions the use of macroeconomic data as input to the SVM classifier to predict the S\&P 500 monthly trend direction [24]. We created a set of data called technical features in the aim of predicting the S\&P 500 monthly trend direction. Using Relative Strength Index, Bollinger Bands, Stochastic, Simple Moving Average, and Momentum, a total of 17 different technical features were constructed. 15 other inputs were constructed using macroeconomic features. The data provided is broken into two periods for training (in-sample period) and test (out-of-sample period). A comparison 
between the efficacy of using macroeconomic and technical features was performed. The next step was to optimize the SVM for both sets of data and compare the results.

\subsubsection{Normalizing}

Because data can be calculated differently and result in different representation to the data, certain data will have high numbers compared to the rest while others may be small. In data mining or machine learning, it is best practice to have the data pre-processed or normalized before the models are built and make use of the data. In this work, we perform normalization by use of the 'zscore', 'normc', and 'normalize' functions with Matlab. Unless turned off, SVM will normalize the data automatically using the zscore method. The way to control this is by setting 'autoscale' from its default value of 'true' to 'false', thus turning off the normalization done internally by the SVM function.

$$
\text { svmStruct = svmtrain(Training, Group, 'autoscale', true); }
$$

Zscore is a very useful statistical tool because it allows us to compare two different values from different normal distributions. Zscore is a function provided by Matlab and computed as follows:

$$
Z(n)=\frac{Y(n)-M}{S}
$$

Where $\mathrm{M}$ is the mean, $\mathrm{S}$ is the standard deviation and $\mathrm{Yn}$ is the value we are normalizing in the vector. A simple example is shown next where we have two 
vectors to normalize, $\mathrm{X}$ and $\mathrm{Y}$. The results show the zscore normalizes each column vector separately and independently.

\begin{tabular}{|c|c|c|c|}
\hline $\mathrm{X}$ & $\mathrm{Y}$ & Zscore $\mathrm{X}$ & Zscore $\mathrm{Y}$ \\
\hline 1 & 2 & -1.1619 & -1.1619 \\
\hline 2 & 4 & -0.3873 & -0.3873 \\
\hline 3 & 6 & 0.3873 & 0.3873 \\
\hline 4 & 8 & 1.1619 & 1.1619 \\
\hline
\end{tabular}

Table 2.1 Example for normalizing simple data with zscore

The function 'normc' normalizes the data to the length of 1 [15]. This function is also provided by Matlab. The normalized vector is computed by:

$$
N(n)=\frac{X(n)}{\|X\|}
$$

Where $\|\mathrm{X}\|$ is the norm of the vector and computed as the following:

$$
\|X\|=\sqrt{\mathrm{X} 1^{2}+\mathrm{X} 2^{2}+\cdots+X n^{2}}
$$

The following table is a simple example for using normc. The same values used for zscore are used here to show the difference. We see normc scales the data to the length of 1 . Using normc, the column data is normalized independently.

\begin{tabular}{|c|c|c|c|}
\hline $\mathrm{X}$ & $\mathrm{Y}$ & Normc $\mathrm{X}$ & Normc $\mathrm{Y}$ \\
\hline 1 & 2 & 0.18257 & 0.18257 \\
\hline 2 & 4 & 0.36515 & 0.36515 \\
\hline
\end{tabular}




\begin{tabular}{|l|l|c|c|}
\hline 3 & 6 & 0.54772 & 0.54772 \\
\hline 4 & 8 & 0.7303 & 0.7303 \\
\hline
\end{tabular}

Table 2.2 Example for normalizing simple data with normc

The function 'normalize' normalizes the data in the vector to become between 0 and 1 and scales the rest of the values appropriately. This function appears not to be provided by Matlab. However, its Matlab implementation is given in an appendix. The normalized vector is computed as the following:

$$
N(n)=\frac{X(n)-X \min }{X \max -X \min }
$$

The same example for normc and zscore is done again to compare the results and show how 'normalize' works.

\begin{tabular}{|c|c|c|c|}
\hline $\mathrm{X}$ & $\mathrm{Y}$ & Normalize $\mathrm{X}$ & Normalize $\mathrm{Y}$ \\
\hline 1 & 2 & 0 & 0 \\
\hline 2 & 4 & 0.33333 & 0.33333 \\
\hline 3 & 6 & 0.66667 & 0.66667 \\
\hline 4 & 8 & 1 & 1 \\
\hline
\end{tabular}

Table 2.3 Example for normalizing simple data with normalize

\subsubsection{Gridsearch}

The classifier's hyperplane can be adjusted based on the model presented by adjusting the parameters that affect the learning algorithm. This is called hyperparameter optimization or model selection and it will ensure that optimizing the model will be done during the in-sample period to not result in overfitting and make sure the out-of-sample classification procedure is not 
affected [16]. There are two parameters for the RBF kernel SVM: C and sigma. A common way of performing this hyperparameter optimization is through gridsearch. The method consists of an in-depth searching through a chosen interval of the parameters. The grid search algorithm is guided by the performance and evaluation of the out-of-sample data. This process can be done by generating a range of values for $\mathrm{C}$ and sigma to search through first. The way used in this paper to generate the values is:

$C=2^{-1}, 2^{-0.9}, 2^{-0.8}, 2^{-0.7}, \ldots 2^{0}, 2^{0.1}, 2^{0.2}, 2^{0.3}, \ldots 2^{1}$ and this is done for sigma as well. Once we find the best parameters, we do another exhaustive search for a very small range where our best parameters are in. The range -1 to 1 is an example to show how it works. This range in this paper was started from 0.001 to $2^{5}$ with increment of 0.001 for the search. Next, we do comprehensive search for all possible pairs of $\mathrm{C}$ and sigma in order to obtain the best classification accuracy of the in-sample data with the optimized parameters [17]. 
Chapter 3 Goals Hypothesis and Evaluation Method

\subsection{Goals}

The main goal of this work was to create a prediction method for the direction of the monthly trend using an appropriate set of data.

The second goal of this work was to learn about the techniques used with Support Vector Machine in computational finance. With all the analysis tools available and the market volatility, it is a hard task to achieve accurate prediction, especially with different kinds of market data that is available. Learning how to classify the data to perform more accurate prediction to the trend direction was an important objective due to unexpected movement of the market.

\subsection{Hypothesis}

The hypothesis of this work was the following:

The financial markets are complex, evolutionary, and non-linear dynamic systems. The market's trend direction can be identified by different type of large data sets. Therefore, predicting and forecasting the market trend is a difficult task. Given the right technical and/or macroeconomic data to a machine learning classifier, such as the Support Vector Machine, it is possible to classify the direction of the market's trend and make accurate investing choices regarding the market and reduce the risk involved. 


\subsection{Evaluation Method}

A simulated predicting system will be constructed using Matlab to test this hypothesis. This system designed will give the option between which features will be used to the SVM classifier and the ability of editing the parameters provided by the classifier in order to maximize the performance. The system will simulate the prediction over a given time period for testing and evaluation. The performance will be measured by the classification accuracy during the insample and out-of-sample. The classification accuracy is the evaluation results of the total number of correctly classified targets compared to the total number of targets. 
Chapter 4: Design

The tool selected to design the prediction simulator was Matlab because of its power and simplicity at the same time. The features used are available mostly in the internet from reliable sources. The prediction simulator was designed to be simple enough and flexible to change the testing period, classifier's kernel, and data selection and reduction.

\subsection{Prediction Model}

In this work, using different type of features and kernels for classification, a model was created in order to find the direction of the trend for the following month.

\subsubsection{Data Construction}

The input data used in this work are separated into two types; macroeconomic and technical data of the S\&P 500 (symbol: SPY). The macroeconomic data was prepared by Amit Goyal and Ivo Welch [18]. The data provided are: DY, EP, DE, SVAR, BM, NTIS, TBL, LTY, LTR, TMS, DFY, DFR and INFL. One extra input was added from the list which is EQ, Equity Premium, as follows: The equity risk premium is the difference between the compound market return and the log return on a risk-free Treasury bill. From the previous statement, we concluded EQ $=$ Compound Return $-\log (1+$ Rfree $)$. The index price was provided by [18] which open, close, high, low were obtained from the 
index price itself. Close price is the index current month's price while open is the previous month's index price. High is defined as the maximum index price over the last 12 months. Low is defined in the same way as high, the lowest index price over the last 12 months. Since we were looking at monthly data with no volume provided, volume indicators were excluded. The data available is from January 1871 to December 2011, which is total of 1680 months of data. The index's monthly price is the closing price of the last trading day of the month. The time period investigated in this work was from June 1938 through December 2010 with the out-of-sample starting in January 1975. Out of 883 trading months used in this work, 439 were used as training data and the remaining 444 used as testing data. The training period was $49.71 \%$ while the testing period was $50.29 \%$. The total features constructed in this work were 27.14 of the total features were macroeconomic while the 13 left were technical features. The construction of the features in Matlab was done through TA-Lib: Technical Analysis Library which is available as an open-source [19]. The macroeconomic inputs are:

1. Dividend price-ratio, DP, is the log of dividends minus the log of prices.

2. Dividend Yield, DY, is the log of dividends minus the log of lagged prices.

3. Earnings are 12-month moving sums of earnings on the S\&P 500 index. Earning Price Ratio, EP, is the log of earnings minus the log of prices.

4. Dividend Payout Ratio, DE, is the log of dividends minus the log of earnings. 
5. Stock Variance, SVAR, is the sum of the monthly return on the S\&P 500.

6. Book-to-Market, BM, is the ratio of book value to market value for the Dow Jones Industrial Average.

7. Net Equity Expansion, NTIS, is the ratio of 12-month moving sums of net issues by NYSE listed stocks divided by the total end-of-year market capitalization of NYSE stocks.

8. Treasury Bills, TBL, is the interest rate on a three-month Treasury bill.

9. Long term yield, LTY, is the long-term government bond yield.

10. Long term return, LTR, is the long-term government bond yield.

11. The Term Spread, TMS, is the long term yield on government bonds minus the Treasury-bill.

12. Default Yield Spread, DFY, is the difference between BAA and AAA-rated corporate bond yields.

13. Default Return Spread, DFR, is the difference between long-term corporate bond and long-term government bond returns.

14. Inflation, INFL, is the Consumer Price Index calculated from All Urban Consumers.

15. DY12 calculated as the difference between log dividends from $P_{t-12}$ to $P_{t}$ at time $\mathrm{t}$.

When constructing the compound return using the given index price from the data in [18] and Yahoo! Finance, there is a mismatch. However, only 9 out of the 1692 data points are not the same when constructing the earnings vector 
to use for training and testing. As a result of this, there is a possible error of $0.53 \%$ or less. The days we found which mismatched are:

\begin{tabular}{|c|c|c|}
\hline Mismatch Date & Price & Previous Month's Price \\
\hline April 1974 & 98.44 & 98.42 \\
\hline April 1979 & 101.76 & 101.59 \\
\hline September 1979 & 109.32 & 109.32 \\
\hline April 1984 & 160.05 & 159.18 \\
\hline January 1986 & 211.78 & 211.28 \\
\hline April 1991 & 375.35 & 375.22 \\
\hline June 1996 & 670.63 & 669.12 \\
\hline February 2006 & 1280.66 & 1280.08 \\
\hline June 2006 & 1270.20 & 1270.09 \\
\hline
\end{tabular}

Table 4.1 Dates of mismatched earnings

From the table 4.1, we notice in those mismatched months, the earnings are so small for each that when we take the difference of the earnings and log of the risk-free, the number we get is negative but during our class labeling, those numbers came out as positive (prior to taking the earnings difference with riskfree).

The technical inputs used are shown in Table 4.2.

\begin{tabular}{|c|c|}
\hline Input \# & Definition \\
\hline 1 & $R S I(c, t)$ \\
\hline 2 & $\frac{c-B \text { Bhigh }}{\text { BBhigh }}$ \\
\hline 3 & $\frac{c-B B \text { low }}{B B l o w}$ \\
\hline 4 & $\% K(t)$ \\
\hline 5 & $\% D(t)$ \\
\hline 6 & $\% K(t)-\% K(t-1)$ \\
\hline 7 & $\% D(t)-\% D(t-1)$ \\
\hline
\end{tabular}




\begin{tabular}{|c|c|}
\hline 8 & $\frac{c(t)-c(t-1)}{c(t-1)}$ \\
\hline 9 & $\frac{c(t)-l(t)}{h(t)-l(t)}$ \\
\hline 10 & $\frac{S M A(c, 10)-S M A(c(t-1), 10)}{S M A(c(t-1), 10)}$ \\
\hline 11 & $\frac{S M A(c, 21)-S M A(c(t-1), 21)}{S M A(c(t-1), 21)}$ \\
\hline 12 & $\frac{S M A(c, 10)-S M A(c(t-1), 21)}{S M A(c(t-1), 21)}$ \\
\hline 13 & $\frac{c(t)-S M A(c, 21)}{S M A(c, 21)}$ \\
\hline 14 & $\frac{c(t)-\min (c, 5)}{\min (c, 5)}$ \\
\hline 15 & $\square(\square)-\square \square \square \square, 5 \square \square \square \square, 5$ \\
\hline 16 & $\frac{S M A(c, 2)-S M A(c, 12)}{S M A(c, 12)}$ \\
\hline 17 & $\frac{c(t)-c(t-12)}{c(t-12)}$ \\
\hline
\end{tabular}

Table 4.2 Set of Technical Indicators Used

Where:

- BBhigh and BBlow are the upper Bollinger Bands and lower Bollinger Bands.

- $\quad c$ is the monthly price of the index.

- $t$ is the time. 


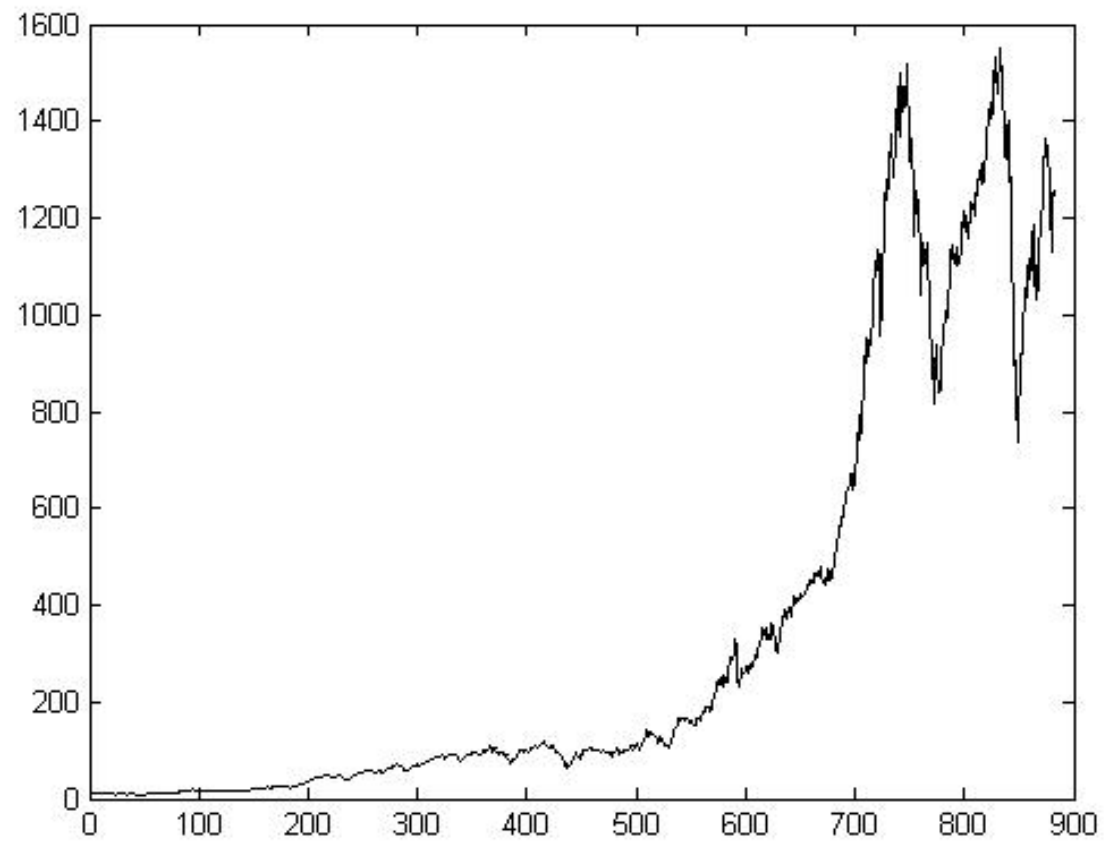

Figure 4.1 S\&P 500 Monthly index over the training and testing period

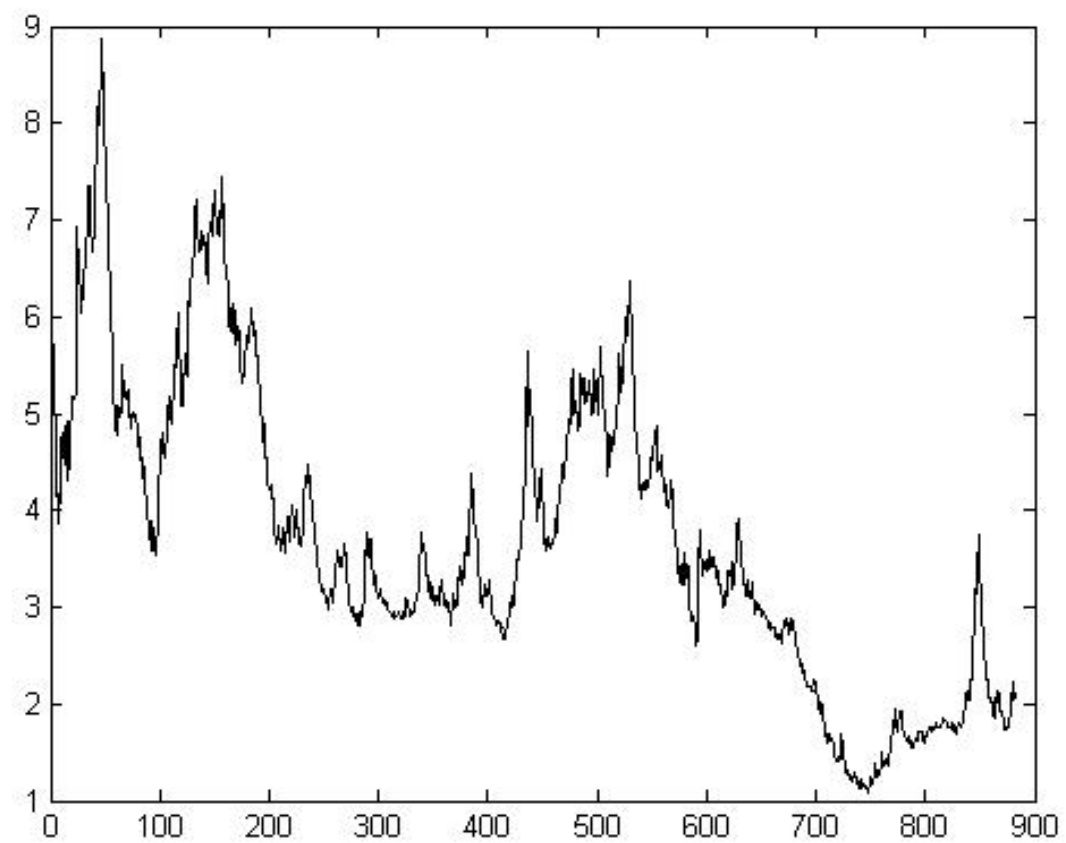

Figure 4.2 S\&P 500 DE movements over the training and testing period 


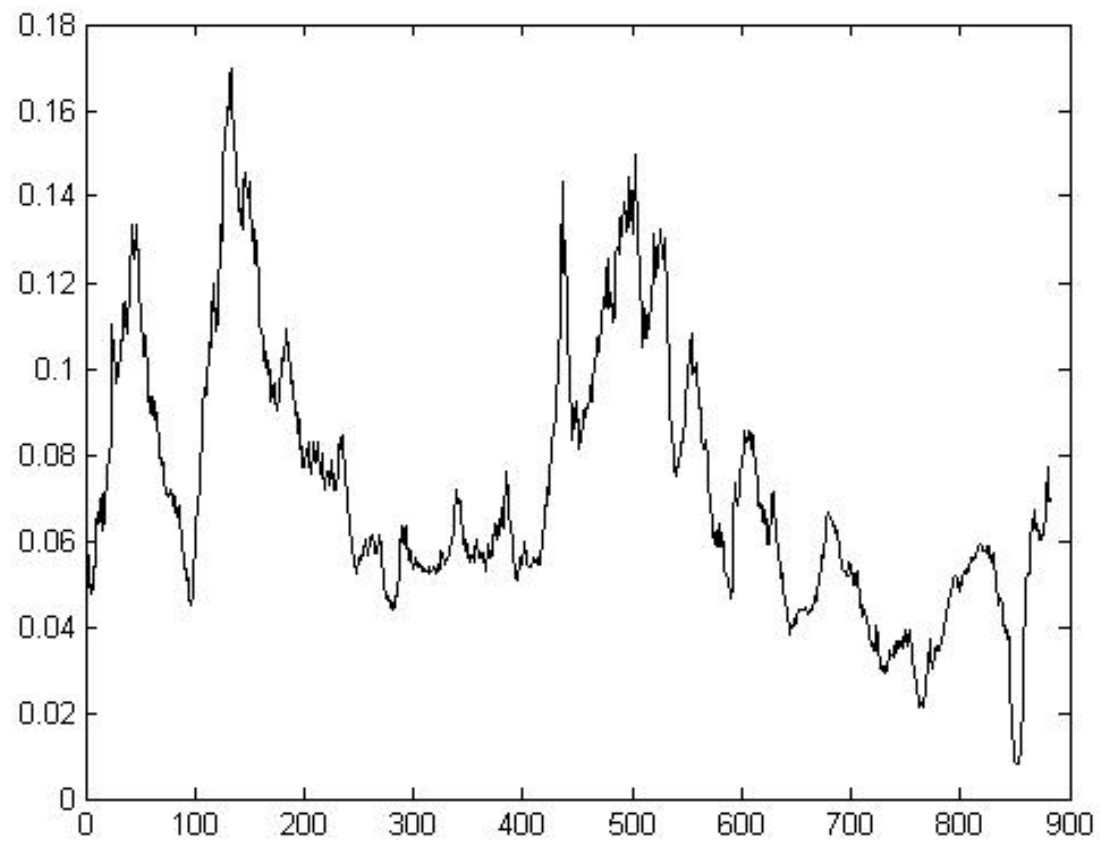

Figure 4.3 S\&P 500 EP movements over the training and testing period

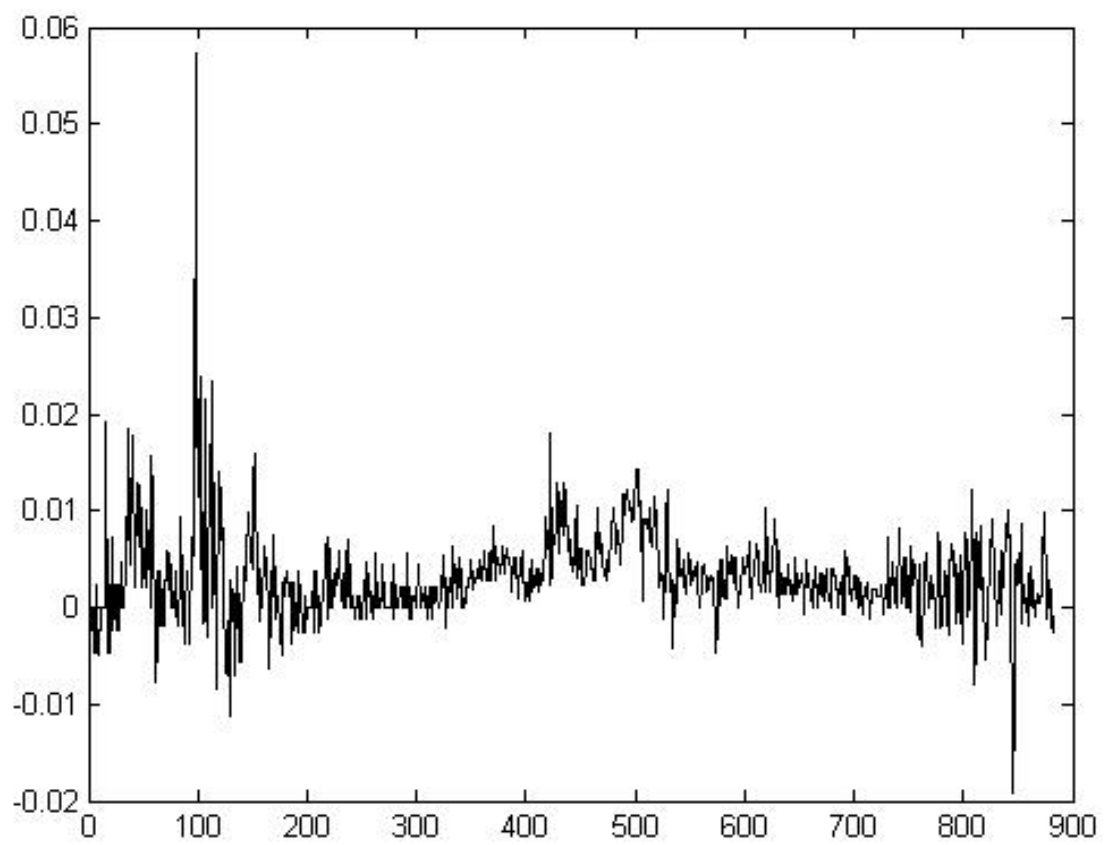

Figure 4.4 S\&P 500 INFL movements over the training and testing period 


\subsubsection{Classification}

The price of the S\&P 500 is a stock market index represented in dollar value. This work deals with S\&P 500 price in time series. A classification method is best to represent the movement of S\&P 500 price in a simplified way. The classification was calculated using a simple difference method; the current month's index price - previous month's index price. The classification then would assign a 1 if next month's index price is higher or equal to the current month and 0 if the price is less than the current month.

\subsubsection{Data Normalization}

It is essential to normalize the data when using a classifier such Support Vector Machine because of features computed may have different value ranges between minimum that is below 0 and maximum to higher than thousands [20]. If features dimensions have fewer variations, it will take less time for SVM to learn and no certain feature that is dominating due to features having fewer dimensions which could impact the behavior over the test data. Normalization is done using the functions 'zscore', 'normc', and 'normalize".

\subsubsection{Proper Data Handling}

Handling the data improperly to the classifier can result in inaccurate classification. When handling all the data at once (in-sample and out-of-sample), the SVM classifier will normalize both periods in a one-time operation. The 
classifier at this point will realize the maximum value in the out-of-sample and this will affect the accuracy of the classification process. First, normalize the insample training data and train the classifier. Normalize the first subset of out-ofsample data as it will be known at this point and the classifier will not be considered looking at the future. Test the classifier for the current out-of-sample data and store the classification result in an array. Next, include the new subset of the out-of-sample data for new normalization (this subset is known at this point) and retrain the classifier for testing. Store the result in the classification array and redo the steps until the out-of-sample data is complete [21].

\subsubsection{Data reduction and selection}

The features used in this work were a total of 15 for macroeconomic and 17 for technical. High dimension problems cause difficulty in classification because of creating many noise features which does not result in contribution to the classification system rather reduces the classification accuracy [22]. Two different methods were used separately in this work in effort to reduce the unnecessary features used while maintaining the robustness of the classification system; Sequential Feature Selection and Rankfeatures. By reducing the features used, the classifier will be dealing with fewer features to learn from.

Reducing the features to the minimum useful while improving the performance in this work was done by looking at the criterion values and the in-sample accuracy 
only, excluding out-of-sample during this procedure. This was done this way because it is not considered looking at future data to take off the data that wasn't useful in out-of-sample.

\subsubsection{Frequent Index's Price Oscillation}

The index's price frequent oscillation in a short period and the ability of the classifier to follow up with changes was an important factor in this work. For example, when in 4 consecutive months, the actual classification records for price movement $1,0,1,0$, the classifier needed to follow those short term price oscillation rather than long term price movement (i.e., 6 months in a row classification is 1 and then another 4 months classification is 0 ).

\subsection{Classifier Data Processing Kernel}

In this work, four different SVM kernels were investigated. The goal was to find the best kernel to classify the data and have a good separation hyperplane between the data sets. Not in all cases the data can be separated, SVM in this case tends to soften the margin in order to separate as much as possible of the data. The kernels used are linear, RBF, quadratic, and polynomial. Finding the best parameters for RBF classifier to soften the margin was done using gridsearch method [23]. 
Chapter 5: Experiments and Results

\subsection{SVM Classification}

We were drawn to do this work by the paper: "Predicting S\&P 500 Returns Using Support Vector Machines: Theory and Empirics" [24] in which a claim was made of achieving an $86 \%$ classification accuracy (we will later show that this claim is unsubstantiated). The model used the given data sets for testing period to predict the direction of the next month's closing price of the S\&P 500. We explore different kernels for the SVM to find the best kernel to classify the data effectively. The features used were macroeconomic and technical. They were each used separately and then combined together with the aim of possibly achieving the highest accuracy in prediction. The same macroeconomic features introduced in [24] were used as an input for the SVM classifier. The technical features used in [25], the same features in Table 4.2, were used as input to the SVM classifier. A combination of both macroeconomic and technical features then was used. The data were normalized using functions,' zscore', 'normc', and 'normalize' in Matlab.

\subsubsection{Macroeconomic Features}

The results for the macroeconomic data test with zscore data normalizing can be seen in Table 5.1. The average in-sample accuracy was $82.92 \%$ for the four different kernels. The average out-of-sample prediction accuracy was $50.92 \%$. The out-of-sample performance for both RBF and quadratic kernels are 
the best with $55.79 \%$ accuracy with the RBF kernel and $54.86 \%$ with quadratic. The polynomial kernel performed the best during the in-sample period but had a lower out-of-sample accuracy with $47.22 \%$ compared to the $100 \%$ accuracy performance during the in-sample period which leads one to conclude that the polynomial was nothing more than a guess work in this case. The RBF kernel was similar in performance to the polynomial in the out-of-sample but has less accuracy during the in-sample period. The RBF has a better success rate compared to the rest of the kernels when considering both the in-sample and out-of-sample accuracy.

\begin{tabular}{|c|c|c|}
\hline Kernel & In-Sample Accuracy \% & Out-Of-Sample Accuracy \% \\
\hline Linear & $59.23 \%$ & $50.46 \%$ \\
\hline RBF & $94.76 \%$ & $55.79 \%$ \\
\hline Polynomial & $97.95 \%$ & $52.08 \%$ \\
\hline Quadratic & $79.73 \%$ & $54.86 \%$ \\
\hline
\end{tabular}

Table 5.1 Results for SVM classifier using Macroeconomic data and zscore normalization

Changing the data normalizing method from 'zscore' to 'normc', the outof-sample accuracy was improved and became overall better than the zscore. The in-sample period accuracy was not as good as with 'zscore' when gaining $59.23 \%$ accuracy for all kernels. The average accuracy for the out-of-sample period was $55 \%$. Classifying resulted in good results using 'normc' during the out-of-sample for all kernels.

\begin{tabular}{|c|c|c|}
\hline Kernel & In-Sample Accuracy \% & Out-Of-Sample Accuracy \% \\
\hline Linear & $59.23 \%$ & $56.02 \%$ \\
\hline RBF & $59.23 \%$ & $55.56 \%$ \\
\hline
\end{tabular}




\begin{tabular}{|c|c|c|}
\hline Polynomial & $59.23 \%$ & $56.02 \%$ \\
\hline Quadratic & $59.23 \%$ & $55.79 \%$ \\
\hline
\end{tabular}

Table 5.2 Results for SVM classifier using Macroeconomic data and normc normalization

The function 'normalize' performed slightly better than 'zscore' during the out-of-sample period but featured less accuracy using quadratic kernel when compared to normalization with zscore in the out-of-sample. The RBF kernel responded well to the normalization with 'normalize' to gain $53.47 \%$ accuracy in the out-of-sample period which was close to the best performing quadratic kernel.

\begin{tabular}{|c|c|c|}
\hline Kernel & In-Sample Accuracy $\%$ & Out-Of-Sample Accuracy $\%$ \\
\hline Linear & $59.68 \%$ & $50.23 \%$ \\
\hline RBF & $66.51 \%$ & $53.47 \%$ \\
\hline Polynomial & $72.67 \%$ & $53.70 \%$ \\
\hline Quadratic & $66.51 \%$ & $53.94 \%$ \\
\hline
\end{tabular}

Table 5.3 Results for SVM classifier using Macroeconomic data with 'normalize' function

Based on our results obtained during the experiment with SVM classification with macroeconomic data and the promotion of the RBF kernel in the paper "Active Learning with Support Vector Machines" [26], we decided to choose RBF kernel and zscore data scaling to investigate the results presented in this paper and compare it with the results in [24]. All of the out-of-sample SVM predictions are presented in Table 5.3. Overall, 241 out of 432 predictions were correct, yielding to $55.79 \%$ accuracy. One thing we notice from the SVM performance was the response to following the long term trend. 


\begin{tabular}{|c|c|c|c|c|c|}
\hline yyyymm & Prediction & Actual & & & \\
\hline 197501 & 1 & 1 & 197803 & 1 & 1 \\
\hline 197502 & 1 & 1 & 197804 & 1 & 1 \\
\hline 197503 & 1 & 1 & 197805 & 1 & 0 \\
\hline 197504 & 1 & 1 & 197806 & 1 & 1 \\
\hline 197505 & 1 & 1 & 197807 & 1 & 1 \\
\hline 197506 & 1 & 0 & 197808 & 1 & 0 \\
\hline 197507 & 1 & 0 & 197809 & 0 & 0 \\
\hline 197508 & 1 & 0 & 197810 & 1 & 1 \\
\hline 197509 & 0 & 1 & 197811 & 1 & 1 \\
\hline 197510 & 1 & 1 & 197812 & 1 & 1 \\
\hline 197511 & 1 & 0 & 197901 & 1 & 0 \\
\hline 197512 & 0 & 1 & 197902 & 1 & 1 \\
\hline 197601 & 1 & 0 & 197903 & 1 & 1 \\
\hline 197602 & 0 & 1 & 197904 & 1 & 0 \\
\hline 197603 & 1 & 0 & 197905 & 1 & 1 \\
\hline 197604 & 0 & 0 & 197906 & 1 & 1 \\
\hline 197605 & 0 & 1 & 197907 & 1 & 1 \\
\hline 197606 & 0 & 0 & 197908 & 1 & 1 \\
\hline 197607 & 0 & 0 & 197909 & 1 & 0 \\
\hline 197608 & 0 & 1 & 197910 & 1 & 1 \\
\hline 197609 & 0 & 0 & 197911 & 1 & 1 \\
\hline 197610 & 0 & 0 & 197912 & 1 & 1 \\
\hline 197611 & 0 & 1 & 198001 & 1 & 0 \\
\hline 197612 & 0 & 0 & 198002 & 1 & 0 \\
\hline 197701 & 0 & 0 & 198003 & 1 & 1 \\
\hline 197702 & 0 & 0 & 198004 & 1 & 1 \\
\hline 197703 & 0 & 1 & 198005 & 1 & 1 \\
\hline 197704 & 0 & 0 & 198006 & 1 & 1 \\
\hline 197705 & 0 & 1 & 198007 & 1 & 1 \\
\hline 197706 & 0 & 0 & 198008 & 1 & 1 \\
\hline 197707 & 0 & 0 & 198009 & 1 & 1 \\
\hline 197708 & 0 & 0 & 198010 & 1 & 1 \\
\hline 197709 & 0 & 0 & 198011 & 0 & 0 \\
\hline 197710 & 0 & 1 & 198012 & 0 & 0 \\
\hline 197711 & 0 & 1 & 198101 & 0 & 1 \\
\hline 197712 & 1 & 0 & 198102 & 1 & 1 \\
\hline 197801 & 1 & 0 & 198103 & 1 & 0 \\
\hline 197802 & 0 & 1 & 198104 & 1 & 0 \\
\hline
\end{tabular}




\begin{tabular}{|c|c|c|c|c|c|}
\hline 198105 & 1 & 0 & 198408 & 0 & 0 \\
\hline 198106 & 1 & 0 & 198409 & 0 & 0 \\
\hline 198107 & 1 & 0 & 198410 & 0 & 0 \\
\hline 198108 & 1 & 0 & 198411 & 0 & 1 \\
\hline 198109 & 1 & 1 & 198412 & 0 & 1 \\
\hline 198110 & 1 & 1 & 198501 & 0 & 1 \\
\hline 198111 & 1 & 0 & 198502 & 0 & 0 \\
\hline 198112 & 1 & 0 & 198503 & 0 & 0 \\
\hline 198201 & 1 & 0 & 198504 & 0 & 1 \\
\hline 198202 & 1 & 0 & 198505 & 0 & 1 \\
\hline 198203 & 1 & 1 & 198506 & 0 & 0 \\
\hline 198204 & 1 & 0 & 198507 & 0 & 0 \\
\hline 198205 & 1 & 0 & 198508 & 0 & 0 \\
\hline 198206 & 1 & 0 & 198509 & 0 & 1 \\
\hline 198207 & 1 & 1 & 198510 & 0 & 1 \\
\hline 198208 & 1 & 1 & 198511 & 0 & 1 \\
\hline 198209 & 1 & 1 & 198512 & 0 & 1 \\
\hline 198210 & 0 & 1 & 198601 & 0 & 1 \\
\hline 198211 & 0 & 1 & 198602 & 0 & 1 \\
\hline 198212 & 0 & 1 & 198603 & 0 & 0 \\
\hline 198301 & 0 & 1 & 198604 & 0 & 1 \\
\hline 198302 & 0 & 1 & 198605 & 0 & 1 \\
\hline 198303 & 0 & 1 & 198606 & 0 & 0 \\
\hline 198304 & 1 & 0 & 198607 & 0 & 1 \\
\hline 198305 & 0 & 1 & 198608 & 0 & 0 \\
\hline 198306 & 0 & 0 & 198609 & 0 & 1 \\
\hline 198307 & 0 & 1 & 198610 & 0 & 1 \\
\hline 198308 & 0 & 1 & 198611 & 1 & 0 \\
\hline 198309 & 0 & 0 & 198612 & 0 & 1 \\
\hline 198310 & 0 & 1 & 198701 & 0 & 1 \\
\hline 198311 & 0 & 0 & 198702 & 1 & 1 \\
\hline 198312 & 0 & 0 & 198703 & 1 & 0 \\
\hline 198401 & 0 & 0 & 198704 & 0 & 1 \\
\hline 198402 & 0 & 1 & 198705 & 1 & 1 \\
\hline 198403 & 0 & 1 & 198706 & 1 & 1 \\
\hline 198404 & 0 & 0 & 198707 & 1 & 1 \\
\hline 198405 & 0 & 1 & 198708 & 1 & 0 \\
\hline 198406 & 0 & 0 & 198709 & 1 & 0 \\
\hline 198407 & 0 & 1 & 198710 & 0 & 0 \\
\hline
\end{tabular}




\begin{tabular}{|c|c|c|c|c|c|}
\hline 198711 & 0 & 1 & 199102 & 1 & 1 \\
\hline 198712 & 0 & 1 & 199103 & 1 & 1 \\
\hline 198801 & 0 & 1 & 199104 & 1 & 1 \\
\hline 198802 & 0 & 0 & 199105 & 1 & 0 \\
\hline 198803 & 0 & 1 & 199106 & 1 & 1 \\
\hline 198804 & 0 & 1 & 199107 & 1 & 1 \\
\hline 198805 & 0 & 1 & 199108 & 1 & 0 \\
\hline 198806 & 0 & 0 & 199109 & 1 & 1 \\
\hline 198807 & 0 & 0 & 199110 & 1 & 0 \\
\hline 198808 & 0 & 1 & 199111 & 1 & 1 \\
\hline 198809 & 0 & 1 & 199112 & 1 & 0 \\
\hline 198810 & 0 & 0 & 199201 & 1 & 1 \\
\hline 198811 & 0 & 1 & 199202 & 1 & 0 \\
\hline 198812 & 0 & 1 & 199203 & 1 & 1 \\
\hline 198901 & 0 & 0 & 199204 & 1 & 1 \\
\hline 198902 & 0 & 1 & 199205 & 1 & 0 \\
\hline 198903 & 0 & 1 & 199206 & 1 & 1 \\
\hline 198904 & 0 & 1 & 199207 & 1 & 0 \\
\hline 198905 & 0 & 0 & 199208 & 1 & 1 \\
\hline 198906 & 0 & 1 & 199209 & 1 & 1 \\
\hline 198907 & 0 & 1 & 199210 & 1 & 1 \\
\hline 198908 & 0 & 0 & 199211 & 1 & 1 \\
\hline 198909 & 0 & 0 & 199212 & 1 & 1 \\
\hline 198910 & 0 & 1 & 199301 & 1 & 1 \\
\hline 198911 & 1 & 1 & 199302 & 1 & 1 \\
\hline 198912 & 1 & 0 & 199303 & 1 & 0 \\
\hline 199001 & 1 & 1 & 199304 & 1 & 1 \\
\hline 199002 & 1 & 1 & 199305 & 1 & 1 \\
\hline 199003 & 1 & 0 & 199306 & 1 & 0 \\
\hline 199004 & 1 & 1 & 199307 & 1 & 1 \\
\hline 199005 & 1 & 0 & 199308 & 1 & 0 \\
\hline 199006 & 1 & 0 & 199309 & 1 & 1 \\
\hline 199007 & 1 & 0 & 199310 & 1 & 0 \\
\hline 199008 & 1 & 0 & 199311 & 1 & 1 \\
\hline 199009 & 1 & 0 & 199312 & 1 & 1 \\
\hline 199010 & 1 & 1 & 199401 & 1 & 0 \\
\hline 199011 & 1 & 1 & 199402 & 1 & 0 \\
\hline 199012 & 1 & 1 & 199403 & 1 & 1 \\
\hline 199101 & 1 & 1 & 199404 & 1 & 1 \\
\hline
\end{tabular}




\begin{tabular}{|c|c|c|c|c|c|}
\hline 199405 & 1 & 0 & 199708 & 1 & 1 \\
\hline 199406 & 1 & 1 & 199709 & 1 & 0 \\
\hline 199407 & 1 & 1 & 199710 & 1 & 1 \\
\hline 199408 & 1 & 0 & 199711 & 1 & 1 \\
\hline 199409 & 1 & 1 & 199712 & 1 & 1 \\
\hline 199410 & 1 & 0 & 199801 & 1 & 1 \\
\hline 199411 & 0 & 1 & 199802 & 1 & 1 \\
\hline 199412 & 0 & 1 & 199803 & 1 & 1 \\
\hline 199501 & 0 & 1 & 199804 & 1 & 0 \\
\hline 199502 & 1 & 1 & 199805 & 1 & 1 \\
\hline 199503 & 0 & 1 & 199806 & 1 & 0 \\
\hline 199504 & 1 & 1 & 199807 & 1 & 0 \\
\hline 199505 & 1 & 1 & 199808 & 1 & 1 \\
\hline 199506 & 1 & 1 & 199809 & 1 & 1 \\
\hline 199507 & 1 & 0 & 199810 & 1 & 1 \\
\hline 199508 & 1 & 1 & 199811 & 1 & 1 \\
\hline 199509 & 1 & 0 & 199812 & 1 & 1 \\
\hline 199510 & 1 & 1 & 199901 & 1 & 0 \\
\hline 199511 & 1 & 1 & 199902 & 1 & 1 \\
\hline 199512 & 1 & 1 & 199903 & 1 & 1 \\
\hline 199601 & 1 & 1 & 199904 & 1 & 0 \\
\hline 199602 & 1 & 1 & 199905 & 1 & 1 \\
\hline 199603 & 1 & 1 & 199906 & 1 & 0 \\
\hline 199604 & 1 & 1 & 199907 & 1 & 0 \\
\hline 199605 & 1 & 1 & 199908 & 1 & 0 \\
\hline 199606 & 1 & 0 & 199909 & 1 & 1 \\
\hline 199607 & 1 & 1 & 199910 & 1 & 1 \\
\hline 199608 & 1 & 1 & 199911 & 1 & 1 \\
\hline 199609 & 1 & 1 & 199912 & 1 & 0 \\
\hline 199610 & 1 & 1 & 200001 & 1 & 0 \\
\hline 199611 & 1 & 0 & 200002 & 1 & 1 \\
\hline 199612 & 1 & 1 & 200003 & 1 & 0 \\
\hline 199701 & 1 & 1 & 200004 & 0 & 0 \\
\hline 199702 & 1 & 0 & 200005 & 0 & 1 \\
\hline 199703 & 1 & 1 & 200006 & 0 & 0 \\
\hline 199704 & 1 & 1 & 200007 & 0 & 1 \\
\hline 199705 & 1 & 1 & 200008 & 0 & 0 \\
\hline 199706 & 1 & 1 & 200009 & 0 & 0 \\
\hline 199707 & 1 & 0 & 200010 & 0 & 0 \\
\hline
\end{tabular}




\begin{tabular}{|c|c|c|c|c|c|}
\hline 200011 & 0 & 1 & 200402 & 1 & 0 \\
\hline 200012 & 0 & 1 & 200403 & 1 & 0 \\
\hline 200101 & 0 & 0 & 200404 & 1 & 1 \\
\hline 200102 & 0 & 0 & 200405 & 1 & 1 \\
\hline 200103 & 0 & 1 & 200406 & 1 & 0 \\
\hline 200104 & 0 & 1 & 200407 & 1 & 1 \\
\hline 200105 & 0 & 0 & 200408 & 1 & 1 \\
\hline 200106 & 0 & 0 & 200409 & 1 & 1 \\
\hline 200107 & 0 & 0 & 200410 & 1 & 1 \\
\hline 200108 & 0 & 0 & 200411 & 1 & 1 \\
\hline 200109 & 0 & 1 & 200412 & 1 & 0 \\
\hline 200110 & 0 & 1 & 200501 & 1 & 1 \\
\hline 200111 & 0 & 1 & 200502 & 1 & 0 \\
\hline 200112 & 1 & 0 & 200503 & 1 & 0 \\
\hline 200201 & 0 & 0 & 200504 & 1 & 1 \\
\hline 200202 & 0 & 1 & 200505 & 1 & 0 \\
\hline 200203 & 0 & 0 & 200506 & 1 & 1 \\
\hline 200204 & 0 & 0 & 200507 & 1 & 0 \\
\hline 200205 & 0 & 0 & 200508 & 1 & 1 \\
\hline 200206 & 0 & 0 & 200509 & 1 & 0 \\
\hline 200207 & 0 & 1 & 200510 & 1 & 1 \\
\hline 200208 & 0 & 0 & 200511 & 1 & 0 \\
\hline 200209 & 0 & 1 & 200512 & 1 & 1 \\
\hline 200210 & 0 & 1 & 200601 & 1 & 1 \\
\hline 200211 & 0 & 0 & 200602 & 1 & 1 \\
\hline 200212 & 0 & 0 & 200603 & 1 & 1 \\
\hline 200301 & 0 & 0 & 200604 & 1 & 0 \\
\hline 200302 & 0 & 1 & 200605 & 1 & 1 \\
\hline 200303 & 0 & 1 & 200606 & 1 & 1 \\
\hline 200304 & 0 & 1 & 200607 & 1 & 1 \\
\hline 200305 & 0 & 1 & 200608 & 1 & 1 \\
\hline 200306 & 1 & 1 & 200609 & 1 & 1 \\
\hline 200307 & 1 & 1 & 200610 & 1 & 1 \\
\hline 200308 & 1 & 0 & 200611 & 1 & 1 \\
\hline 200309 & 1 & 1 & 200612 & 1 & 1 \\
\hline 200310 & 1 & 1 & 200701 & 1 & 0 \\
\hline 200311 & 1 & 1 & 200702 & 1 & 1 \\
\hline 200312 & 1 & 1 & 200703 & 1 & 1 \\
\hline 200401 & 1 & 1 & 200704 & 1 & 1 \\
\hline
\end{tabular}




\begin{tabular}{|c|c|c|c|c|c|}
\hline 200705 & 1 & 0 & 200909 & 1 & 0 \\
\hline 200706 & 1 & 0 & 200910 & 0 & 1 \\
\hline 200707 & 1 & 1 & 200911 & 1 & 1 \\
\hline 200708 & 1 & 1 & 200912 & 1 & 0 \\
\hline 200709 & 1 & 1 & 201001 & 1 & 1 \\
\hline 200710 & 1 & 0 & 201002 & 1 & 1 \\
\hline 200711 & 1 & 0 & 201003 & 1 & 1 \\
\hline 200712 & 1 & 0 & 201004 & 1 & 0 \\
\hline 200801 & 1 & 0 & 201005 & 1 & 0 \\
\hline 200802 & 1 & 0 & 201006 & 1 & 1 \\
\hline 200803 & 1 & 1 & 201007 & 1 & 0 \\
\hline 200804 & 1 & 1 & 201008 & 1 & 1 \\
\hline 200805 & 1 & 0 & 201009 & 1 & 1 \\
\hline 200806 & 1 & 0 & 201010 & 1 & 0 \\
\hline 200807 & 1 & 1 & 201011 & 1 & 1 \\
\hline 200808 & 1 & 0 & 201012 & 1 & 1 \\
\hline 200809 & 0 & 0 & & & \\
\hline 200810 & 0 & 0 & & & \\
\hline 200811 & 0 & 1 & & & \\
\hline 200812 & 1 & 0 & & & \\
\hline 200901 & 0 & 0 & & & \\
\hline 200902 & 0 & 1 & & & \\
\hline 200903 & 0 & 1 & & & \\
\hline 200904 & 1 & 1 & & & \\
\hline 200905 & 1 & 1 & & & \\
\hline 200906 & 1 & 1 & & & \\
\hline 200907 & 1 & 1 & & & \\
\hline 200908 & 1 & 1 & & & \\
\hline
\end{tabular}

Table 5.4 Out-of-sample SVM predictions vs. actual realization 


\subsubsection{Technical Features}

The work in [24] indicates the use of macroeconomic variables will tend to lead to superior prediction rates compared to the use of technical features. The next step in this work was to substitute the macroeconomic features with the technical features presented in [25]. The first test was to use technical features with zscore. The accuracy of the classification can be seen in Table 5.5.

\begin{tabular}{|c|c|c|}
\hline Kernel & In-Sample Accuracy \% & Out-Of-Sample Accuracy \% \\
\hline Linear & $60.59 \%$ & $51.16 \%$ \\
\hline RBF & $95.90 \%$ & $59.26 \%$ \\
\hline Polynomial & $99.32 \%$ & $49.77 \%$ \\
\hline Quadratic & $77.68 \%$ & $49.54 \%$ \\
\hline
\end{tabular}

Table 5.5 Results for SVM classifier using technical features and zscore normalization

The RBF and Polynomial kernels performed better during the in-sample with technical features as well. The polynomial kernel was able to perform the best during the in-sample period but not as well during out-of-sample to result in classification accuracy below $50 \%$. Compared to the macroeconomic features, the classification accuracy of the RBF was the best at $59.26 \%$ to outperform the best performance made using macroeconomic features. The quadratic kernel had better results using the macroeconomic features compared to using technical features during the out-of-sample test.

\begin{tabular}{|c|c|c|}
\hline Kernel & In-Sample Accuracy $\%$ & Out-Of-Sample Accuracy $\%$ \\
\hline Linear & $56.04 \%$ & $48.15 \%$ \\
\hline RBF & $56.04 \%$ & $48.15 \%$ \\
\hline Polynomial & $55.58 \%$ & $46.99 \%$ \\
\hline
\end{tabular}




\begin{tabular}{|l|l|l|}
\hline Quadratic & $56.04 \%$ & $46.99 \%$ \\
\hline
\end{tabular}

Table 5.6 Results for SVM classifier using technical features and normc normalization

Next, the data scaling was tested using normc function and the results can be seen in Table 5.6. Normc shows very similar performance during the insample and out-of-sample periods. Classification with technical data showed similar behavior when using the normc to classifying with macroeconomic features. All kernels had similar results for the in-sample and out-of-sample

The next step was to perform data normalization use the 'normalize' function. The polynomial kernel made a noticeable improvement in comparison with its performance using the normc and zscore functions. It outperformed the normc function and was able to achieve higher than $50 \%$ classification accuracy during the out-of-sample test period. From Table 5.7, the best performing kernel was the RBF with $55.56 \%$ accuracy during the out-of-sample period.

\begin{tabular}{|c|c|c|}
\hline Kernel & In-Sample Accuracy $\%$ & Out-Of-Sample Accuracy $\%$ \\
\hline Linear & $61.73 \%$ & $52.55 \%$ \\
\hline RBF & $61.96 \%$ & $55.56 \%$ \\
\hline Polynomial & $69.25 \%$ & $51.39 \%$ \\
\hline Quadratic & $64.24 \%$ & $54.63 \%$ \\
\hline
\end{tabular}

Table 5.7 Results for SVM classifier using technical features and normalize function

The classification results using zscore are still the best even when using technical features which are simple to obtain compared to macroeconomic data. Looking closely at classification results of the best overall performing kernel with technical features, the RBF with zscore data normalizing, the classifier predicted 
256 out-of-sample data correctly out of 432 . The classifier with technical features responded well to the short trend oscillation and making classification accuracy close to $60 \%$. The following table shows detailed results of classification with technical features and RBF kernel:

\begin{tabular}{|c|c|c|c|c|c|}
\hline yyyymm & Prediction & Actual & & & \\
\hline 197501 & 1 & 1 & 197707 & 0 & 0 \\
\hline 197502 & 1 & 1 & 197708 & 1 & 0 \\
\hline 197503 & 1 & 1 & 197709 & 0 & 0 \\
\hline 197504 & 1 & 1 & 197710 & 0 & 1 \\
\hline 197505 & 1 & 1 & 197711 & 1 & 1 \\
\hline 197506 & 1 & 0 & 197712 & 1 & 0 \\
\hline 197507 & 1 & 0 & 197801 & 0 & 0 \\
\hline 197508 & 1 & 0 & 197802 & 0 & 1 \\
\hline 197509 & 1 & 1 & 197803 & 1 & 1 \\
\hline 197510 & 0 & 1 & 197804 & 1 & 1 \\
\hline 197511 & 0 & 0 & 197805 & 1 & 0 \\
\hline 197512 & 1 & 1 & 197806 & 0 & 1 \\
\hline 197601 & 1 & 0 & 197807 & 1 & 1 \\
\hline 197602 & 1 & 1 & 197808 & 1 & 0 \\
\hline 197603 & 1 & 0 & 197809 & 1 & 0 \\
\hline 197604 & 1 & 0 & 197810 & 1 & 1 \\
\hline 197605 & 1 & 1 & 197811 & 0 & 1 \\
\hline 197606 & 1 & 0 & 197812 & 1 & 1 \\
\hline 197607 & 1 & 0 & 197901 & 0 & 0 \\
\hline 197608 & 1 & 1 & 197902 & 1 & 1 \\
\hline 197609 & 1 & 0 & 197903 & 0 & 1 \\
\hline 197610 & 1 & 0 & 197904 & 1 & 0 \\
\hline 197611 & 1 & 1 & 197905 & 1 & 1 \\
\hline 197612 & 1 & 0 & 197906 & 1 & 1 \\
\hline 197701 & 1 & 0 & 197907 & 1 & 1 \\
\hline 197702 & 0 & 0 & 197908 & 0 & 1 \\
\hline 197703 & 0 & 1 & 197909 & 1 & 0 \\
\hline 197704 & 1 & 0 & 197910 & 1 & 1 \\
\hline 197705 & 1 & 1 & 197911 & 0 & 1 \\
\hline 197706 & 0 & 0 & 197912 & 0 & 1 \\
\hline
\end{tabular}




\begin{tabular}{|c|c|c|c|c|c|}
\hline 198001 & 1 & 0 & 198304 & 1 & 0 \\
\hline 198002 & 0 & 0 & 198305 & 0 & 1 \\
\hline 198003 & 1 & 1 & 198306 & 1 & 0 \\
\hline 198004 & 1 & 1 & 198307 & 1 & 1 \\
\hline 198005 & 1 & 1 & 198308 & 1 & 1 \\
\hline 198006 & 1 & 1 & 198309 & 1 & 0 \\
\hline 198007 & 1 & 1 & 198310 & 0 & 1 \\
\hline 198008 & 0 & 1 & 198311 & 1 & 0 \\
\hline 198009 & 0 & 1 & 198312 & 0 & 0 \\
\hline 198010 & 1 & 1 & 198401 & 0 & 0 \\
\hline 198011 & 1 & 0 & 198402 & 1 & 1 \\
\hline 198012 & 1 & 0 & 198403 & 0 & 1 \\
\hline 198101 & 1 & 1 & 198404 & 1 & 0 \\
\hline 198102 & 1 & 1 & 198405 & 1 & 1 \\
\hline 198103 & 1 & 0 & 198406 & 0 & 0 \\
\hline 198104 & 0 & 0 & 198407 & 1 & 1 \\
\hline 198105 & 0 & 0 & 198408 & 1 & 0 \\
\hline 198106 & 0 & 0 & 198409 & 1 & 0 \\
\hline 198107 & 0 & 0 & 198410 & 0 & 0 \\
\hline 198108 & 0 & 0 & 198411 & 1 & 1 \\
\hline 198109 & 1 & 1 & 198412 & 1 & 1 \\
\hline 198110 & 1 & 1 & 198501 & 1 & 1 \\
\hline 198111 & 0 & 0 & 198502 & 1 & 0 \\
\hline 198112 & 1 & 0 & 198503 & 0 & 0 \\
\hline 198201 & 0 & 0 & 198504 & 0 & 1 \\
\hline 198202 & 1 & 0 & 198505 & 1 & 1 \\
\hline 198203 & 1 & 1 & 198506 & 0 & 0 \\
\hline 198204 & 1 & 0 & 198507 & 0 & 0 \\
\hline 198205 & 0 & 0 & 198508 & 1 & 0 \\
\hline 198206 & 1 & 0 & 198509 & 0 & 1 \\
\hline 198207 & 0 & 1 & 198510 & 1 & 1 \\
\hline 198208 & 1 & 1 & 198511 & 1 & 1 \\
\hline 198209 & 0 & 1 & 198512 & 1 & 1 \\
\hline 198210 & 1 & 1 & 198601 & 1 & 1 \\
\hline 198211 & 1 & 1 & 198602 & 1 & 1 \\
\hline 198212 & 1 & 1 & 198603 & 0 & 0 \\
\hline 198301 & 1 & 1 & 198604 & 0 & 1 \\
\hline 198302 & 1 & 1 & 198605 & 0 & 1 \\
\hline 198303 & 1 & 1 & 198606 & 0 & 0 \\
\hline
\end{tabular}




\begin{tabular}{|c|c|c|c|c|c|}
\hline 198607 & 1 & 1 & 198910 & 1 & 1 \\
\hline 198608 & 0 & 0 & 198911 & 1 & 1 \\
\hline 198609 & 1 & 1 & 198912 & 1 & 0 \\
\hline 198610 & 1 & 1 & 199001 & 1 & 1 \\
\hline 198611 & 0 & 0 & 199002 & 0 & 1 \\
\hline 198612 & 0 & 1 & 199003 & 1 & 0 \\
\hline 198701 & 1 & 1 & 199004 & 0 & 1 \\
\hline 198702 & 1 & 1 & 199005 & 1 & 0 \\
\hline 198703 & 1 & 0 & 199006 & 1 & 0 \\
\hline 198704 & 1 & 1 & 199007 & 0 & 0 \\
\hline 198705 & 1 & 1 & 199008 & 0 & 0 \\
\hline 198706 & 0 & 1 & 199009 & 0 & 0 \\
\hline 198707 & 0 & 1 & 199010 & 1 & 1 \\
\hline 198708 & 0 & 0 & 199011 & 1 & 1 \\
\hline 198709 & 1 & 0 & 199012 & 0 & 1 \\
\hline 198710 & 1 & 0 & 199101 & 1 & 1 \\
\hline 198711 & 1 & 1 & 199102 & 1 & 1 \\
\hline 198712 & 1 & 1 & 199103 & 1 & 1 \\
\hline 198801 & 1 & 1 & 199104 & 1 & 1 \\
\hline 198802 & 1 & 0 & 199105 & 1 & 0 \\
\hline 198803 & 1 & 1 & 199106 & 1 & 1 \\
\hline 198804 & 0 & 1 & 199107 & 1 & 1 \\
\hline 198805 & 0 & 1 & 199108 & 0 & 0 \\
\hline 198806 & 1 & 0 & 199109 & 1 & 1 \\
\hline 198807 & 0 & 0 & 199110 & 0 & 0 \\
\hline 198808 & 0 & 1 & 199111 & 1 & 1 \\
\hline 198809 & 1 & 1 & 199112 & 1 & 0 \\
\hline 198810 & 1 & 0 & 199201 & 0 & 1 \\
\hline 198811 & 1 & 1 & 199202 & 0 & 0 \\
\hline 198812 & 0 & 1 & 199203 & 0 & 1 \\
\hline 198901 & 1 & 0 & 199204 & 1 & 1 \\
\hline 198902 & 1 & 1 & 199205 & 1 & 0 \\
\hline 198903 & 0 & 1 & 199206 & 1 & 1 \\
\hline 198904 & 0 & 1 & 199207 & 0 & 0 \\
\hline 198905 & 1 & 0 & 199208 & 1 & 1 \\
\hline 198906 & 0 & 1 & 199209 & 1 & 1 \\
\hline 198907 & 1 & 1 & 199210 & 1 & 1 \\
\hline 198908 & 1 & 0 & 199211 & 1 & 1 \\
\hline 198909 & 0 & 0 & 199212 & 1 & 1 \\
\hline
\end{tabular}




\begin{tabular}{|c|c|c|c|c|c|}
\hline 199301 & 1 & 1 & 199604 & 1 & 1 \\
\hline 199302 & 1 & 1 & 199605 & 1 & 1 \\
\hline 199303 & 1 & 0 & 199606 & 1 & 0 \\
\hline 199304 & 0 & 1 & 199607 & 1 & 1 \\
\hline 199305 & 1 & 1 & 199608 & 1 & 1 \\
\hline 199306 & 1 & 0 & 199609 & 0 & 1 \\
\hline 199307 & 1 & 1 & 199610 & 0 & 1 \\
\hline 199308 & 0 & 0 & 199611 & 1 & 0 \\
\hline 199309 & 1 & 1 & 199612 & 1 & 1 \\
\hline 199310 & 1 & 0 & 199701 & 1 & 1 \\
\hline 199311 & 1 & 1 & 199702 & 1 & 0 \\
\hline 199312 & 1 & 1 & 199703 & 1 & 1 \\
\hline 199401 & 0 & 0 & 199704 & 1 & 1 \\
\hline 199402 & 1 & 0 & 199705 & 1 & 1 \\
\hline 199403 & 0 & 1 & 199706 & 1 & 1 \\
\hline 199404 & 1 & 1 & 199707 & 1 & 0 \\
\hline 199405 & 1 & 0 & 199708 & 1 & 1 \\
\hline 199406 & 0 & 1 & 199709 & 0 & 0 \\
\hline 199407 & 0 & 1 & 199710 & 1 & 1 \\
\hline 199408 & 1 & 0 & 199711 & 0 & 1 \\
\hline 199409 & 1 & 1 & 199712 & 0 & 1 \\
\hline 199410 & 0 & 0 & 199801 & 0 & 1 \\
\hline 199411 & 0 & 1 & 199802 & 1 & 1 \\
\hline 199412 & 0 & 1 & 199803 & 1 & 1 \\
\hline 199501 & 0 & 1 & 199804 & 0 & 0 \\
\hline 199502 & 1 & 1 & 199805 & 1 & 1 \\
\hline 199503 & 1 & 1 & 199806 & 0 & 0 \\
\hline 199504 & 0 & 1 & 199807 & 1 & 0 \\
\hline 199505 & 0 & 1 & 199808 & 1 & 1 \\
\hline 199506 & 1 & 1 & 199809 & 1 & 1 \\
\hline 199507 & 1 & 0 & 199810 & 1 & 1 \\
\hline 199508 & 0 & 1 & 199811 & 1 & 1 \\
\hline 199509 & 0 & 0 & 199812 & 1 & 1 \\
\hline 199510 & 1 & 1 & 199901 & 1 & 0 \\
\hline 199511 & 0 & 1 & 199902 & 1 & 1 \\
\hline 199512 & 0 & 1 & 199903 & 1 & 1 \\
\hline 199601 & 1 & 1 & 199904 & 1 & 0 \\
\hline 199602 & 1 & 1 & 199905 & 1 & 1 \\
\hline 199603 & 1 & 1 & 199906 & 1 & 0 \\
\hline
\end{tabular}




\begin{tabular}{|c|c|c|c|c|c|}
\hline 199907 & 0 & 0 & 200210 & 1 & 1 \\
\hline 199908 & 0 & 0 & 200211 & 1 & 0 \\
\hline 199909 & 1 & 1 & 200212 & 1 & 0 \\
\hline 199910 & 1 & 1 & 200301 & 0 & 0 \\
\hline 199911 & 0 & 1 & 200302 & 0 & 1 \\
\hline 199912 & 1 & 0 & 200303 & 1 & 1 \\
\hline 200001 & 1 & 0 & 200304 & 1 & 1 \\
\hline 200002 & 1 & 1 & 200305 & 1 & 1 \\
\hline 200003 & 1 & 0 & 200306 & 1 & 1 \\
\hline 200004 & 0 & 0 & 200307 & 1 & 1 \\
\hline 200005 & 1 & 1 & 200308 & 1 & 0 \\
\hline 200006 & 0 & 0 & 200309 & 1 & 1 \\
\hline 200007 & 1 & 1 & 200310 & 0 & 1 \\
\hline 200008 & 0 & 0 & 200311 & 1 & 1 \\
\hline 200009 & 1 & 0 & 200312 & 0 & 1 \\
\hline 200010 & 0 & 0 & 200401 & 0 & 1 \\
\hline 200011 & 0 & 1 & 200402 & 0 & 0 \\
\hline 200012 & 0 & 1 & 200403 & 1 & 0 \\
\hline 200101 & 1 & 0 & 200404 & 1 & 1 \\
\hline 200102 & 1 & 0 & 200405 & 1 & 1 \\
\hline 200103 & 1 & 1 & 200406 & 0 & 0 \\
\hline 200104 & 1 & 1 & 200407 & 1 & 1 \\
\hline 200105 & 0 & 0 & 200408 & 1 & 1 \\
\hline 200106 & 0 & 0 & 200409 & 1 & 1 \\
\hline 200107 & 0 & 0 & 200410 & 1 & 1 \\
\hline 200108 & 0 & 0 & 200411 & 0 & 1 \\
\hline 200109 & 0 & 1 & 200412 & 0 & 0 \\
\hline 200110 & 0 & 1 & 200501 & 1 & 1 \\
\hline 200111 & 1 & 1 & 200502 & 0 & 0 \\
\hline 200112 & 1 & 0 & 200503 & 1 & 0 \\
\hline 200201 & 0 & 0 & 200504 & 0 & 1 \\
\hline 200202 & 0 & 1 & 200505 & 1 & 0 \\
\hline 200203 & 1 & 0 & 200506 & 1 & 1 \\
\hline 200204 & 0 & 0 & 200507 & 1 & 0 \\
\hline 200205 & 1 & 0 & 200508 & 1 & 1 \\
\hline 200206 & 1 & 0 & 200509 & 1 & 0 \\
\hline 200207 & 1 & 1 & 200510 & 0 & 1 \\
\hline 200208 & 1 & 0 & 200511 & 1 & 0 \\
\hline 200209 & 1 & 1 & 200512 & 1 & 1 \\
\hline
\end{tabular}




\begin{tabular}{|c|c|c|c|c|c|}
\hline 200601 & 1 & 1 & 200808 & 1 & 0 \\
\hline 200602 & 1 & 1 & 200809 & 0 & 0 \\
\hline 200603 & 1 & 1 & 200810 & 1 & 0 \\
\hline 200604 & 0 & 0 & 200811 & 1 & 1 \\
\hline 200605 & 0 & 1 & 200812 & 1 & 0 \\
\hline 200606 & 0 & 1 & 200901 & 1 & 0 \\
\hline 200607 & 1 & 1 & 200902 & 1 & 1 \\
\hline 200608 & 0 & 1 & 200903 & 1 & 1 \\
\hline 200609 & 1 & 1 & 200904 & 1 & 1 \\
\hline 200610 & 0 & 1 & 200905 & 1 & 1 \\
\hline 200611 & 0 & 1 & 200906 & 1 & 1 \\
\hline 200612 & 0 & 1 & 200907 & 1 & 1 \\
\hline 200701 & 0 & 0 & 200908 & 1 & 1 \\
\hline 200702 & 0 & 1 & 200909 & 1 & 0 \\
\hline 200703 & 1 & 1 & 200910 & 1 & 1 \\
\hline 200704 & 0 & 1 & 200911 & 0 & 1 \\
\hline 200705 & 0 & 0 & 200912 & 1 & 0 \\
\hline 200706 & 0 & 0 & 201001 & 1 & 1 \\
\hline 200707 & 1 & 1 & 201002 & 1 & 1 \\
\hline 200708 & 1 & 1 & 201003 & 1 & 1 \\
\hline 200709 & 1 & 1 & 201004 & 1 & 0 \\
\hline 200710 & 0 & 0 & 201005 & 1 & 0 \\
\hline 200711 & 0 & 0 & 201006 & 1 & 1 \\
\hline 200712 & 0 & 0 & 201007 & 1 & 0 \\
\hline 200801 & 1 & 0 & 201008 & 1 & 1 \\
\hline 200802 & 1 & 0 & 201009 & 1 & 1 \\
\hline 200803 & 1 & 1 & 201010 & 1 & 0 \\
\hline 200804 & 1 & 1 & 201011 & 1 & 1 \\
\hline 200805 & 0 & 0 & 201012 & 1 & 1 \\
\hline 200806 & 1 & 0 & & & \\
\hline 200807 & 1 & 1 & & & \\
\hline
\end{tabular}

Table 5.8 Technical Out-of-sample SVM predictions vs. actual realization 


\subsection{SVM RBF Kernel Parameters Selection and Optimization}

The next step was to adjust the width of SVM RBF classifier using parameters $\mathrm{C}$ and sigma which will control the margin of classification. One way to do this is with a grid search method as mentioned in the previous chapters and papers [27] and [28]. The goal of this search was to find the best pair of parameters during the in-sample period to maximize the out-of-sample accuracy as much as possible. The default values for both parameters in Matlab were "1" for parameters, $C$ and sigma. At the end of our work, the parameter $C$ was not modified, because once we optimized for the best value of sigma for the RBF kernel, if we were to modify the parameter $C$ further, it would have resulted in a softer margin for classification. This will result in slow response of the classifier (i.e. keeping the classification rate at 1 or 0 for a long period of time).

From Figure 5.1 we notice during optimizing the RBF sigma parameter, setting the sigma parameter slightly higher than the default, the higher the accuracy we get. The optimal sigma parameter using macroeconomic features was Sigma $=0.45$ which increased the performance for the out-of-sample period from $55.79 \%$ to $59.25 \%$ with zscore data normalizing. 


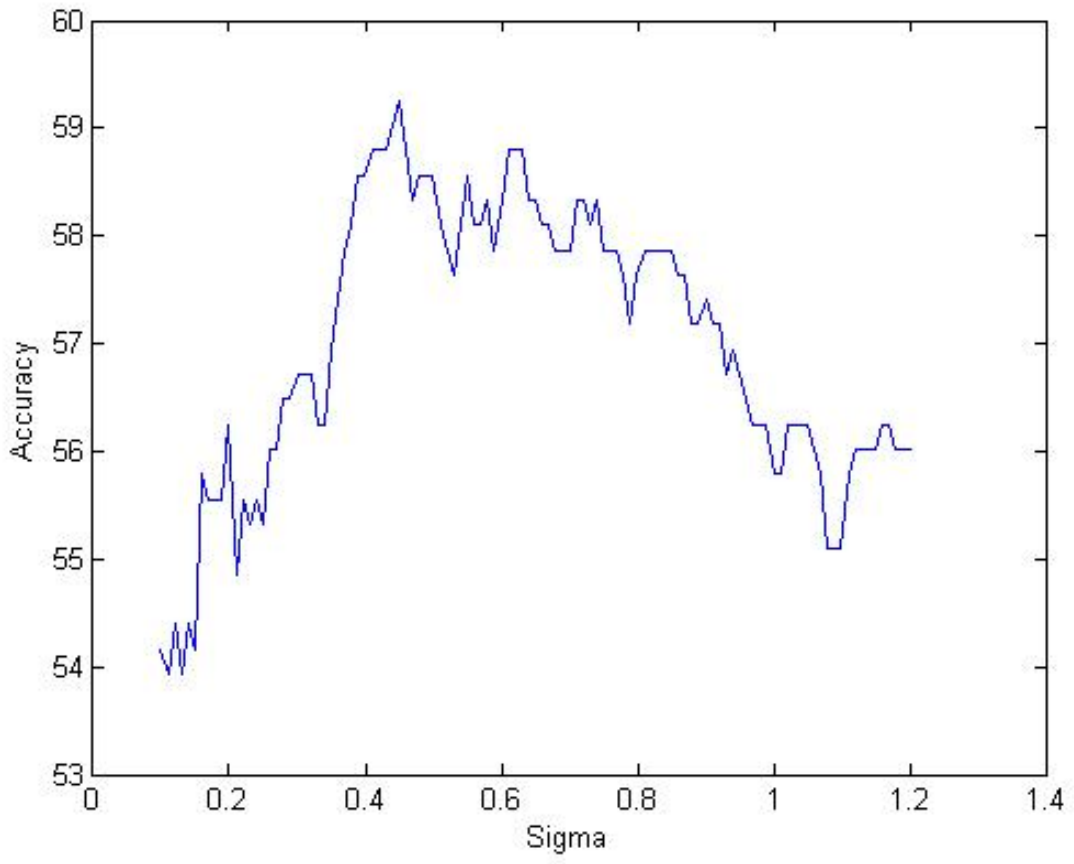

Figure 5.1 Out-of-sample accuracy using macroeconomic data vs. parameters change

Optimizing the RBF parameters to the classifier using technical features input showed noticeable improvement. From figure 5.2, the parameter sigma increased the out-of-sample accuracy to $62.03 \%$ with the parameter Sigma $=$ 0.425 . This change increased the out-of-sample classification accuracy by $2.77 \%$ from $59.26 \%$ to $62.03 \%$. The data normalization method used was zscore. Technical features made a better out-of-sample accuracy with the optimized parameters than macroeconomic features. 


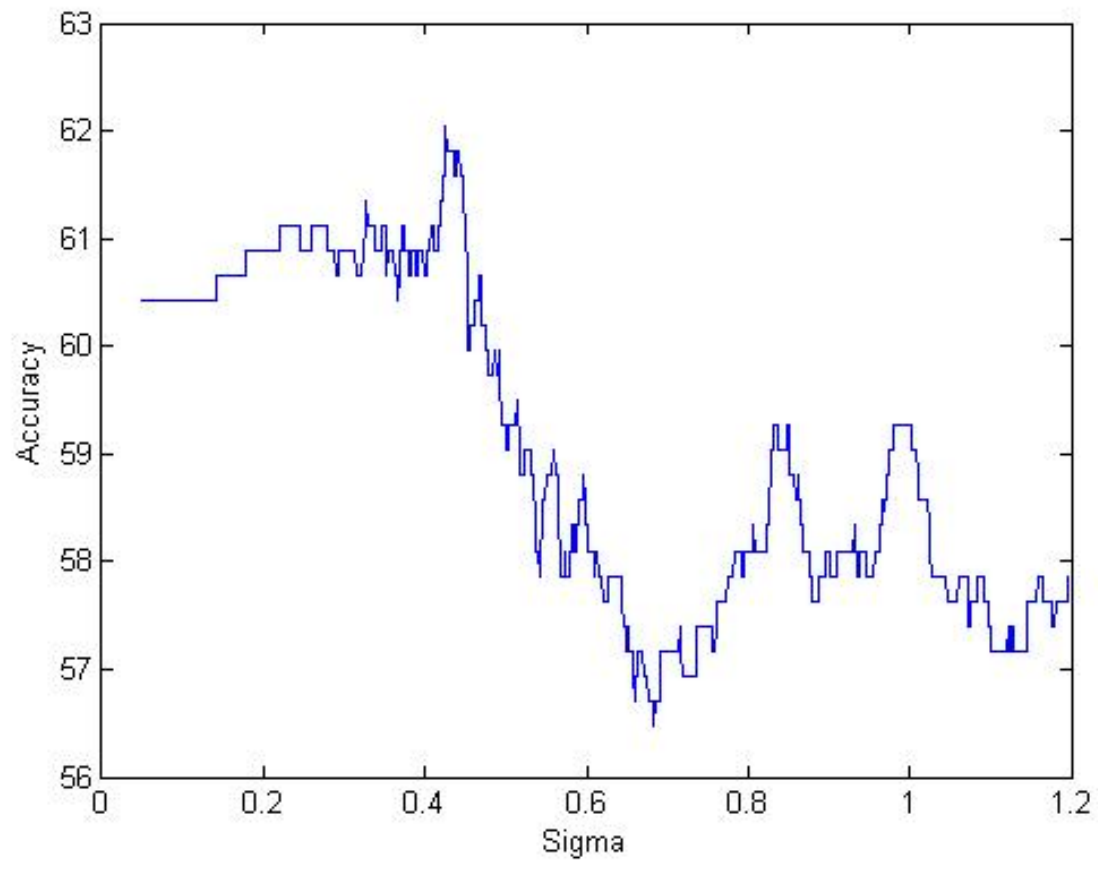

Figure 5.2 Out-of-sample accuracy using technical data vs. parameters change

\subsection{Feature Selection and Dimensionality Reduction}

The goal of feature reduction was to reduce the problem dimensionality by reducing the dataset in order to exclude irrelevant features and at the same time not have the dataset too small to cause the classifier to be over fit [29].

Sequential Feature Selection and Rankfeatures tests were performed in order to find the minimum possible features needed. The tests were performed to linear and RBF kernels since they are used mostly in this kind of work. 


\subsubsection{Sequential Feature Selection}

\subsubsection{Sequential Feature Reduction with Macroeconomic Features}

Linear Kernel:

Sequential Feature Selection was performed using the most widely used two kernels in this sort of application from section 5.1 to both, macroeconomic and technical features. Table 5.9 shows the features significance using linear kernel and 'zscore' data scaling.

\begin{tabular}{|c|c|}
\hline $\begin{array}{c}\text { Feature } \\
\text { Rank }\end{array}$ & $\begin{array}{c}\text { Feature } \\
\#\end{array}$ \\
\hline 1 & 14 \\
\hline 2 & 4 \\
\hline 3 & 5 \\
\hline 4 & 7 \\
\hline 5 & 1 \\
\hline 6 & 3 \\
\hline 7 & 15 \\
\hline 8 & 2 \\
\hline 9 & 8 \\
\hline 10 & 10 \\
\hline 11 & 6 \\
\hline 12 & 9 \\
\hline 13 & 13 \\
\hline 14 & 11 \\
\hline 15 & 12 \\
\hline
\end{tabular}

Table 5.9 Macroeconomic Features Significance Ranking using Sequential Feature and linear kernel

Table 5.10 shows the results of Sequential Feature Selection test using macroeconomic data, zscore data scaling, and linear kernel. 


\begin{tabular}{|c|c|}
\hline \# of Features & Out-of-Sample Accuracy \\
\hline 1 & $60.42 \%$ \\
\hline 2 & $40.50 \%$ \\
\hline 3 & $40.50 \%$ \\
\hline 4 & $40.27 \%$ \\
\hline 5 & $42.13 \%$ \\
\hline 6 & $46.29 \%$ \\
\hline 7 & $45.83 \%$ \\
\hline 8 & $45.83 \%$ \\
\hline 9 & $53.93 \%$ \\
\hline 10 & $53.24 \%$ \\
\hline 11 & $51.15 \%$ \\
\hline 12 & $50.92 \%$ \\
\hline 13 & $50.69 \%$ \\
\hline 14 & $51.38 \%$ \\
\hline 15 & $50.46 \%$ \\
\hline
\end{tabular}

Table 5.10 Sequential Feature Selection for macroeconomic data with Linear Kernel

From the Table 5.10, we notice that only one feature, namely inflation, out of 15 resulted in the best performance for linear kernel.

RBF Kernel:

Using an RBF kernel gave us different results from the linear kernel. Using 'zscore' when reducing the number of features, we were able to obtain the best result for macroeconomic with sequential feature reduction where the best out-of-sample accuracy was $58.33 \%$ using 3 features only (TBL, DFR, and DFY) as seen in Table 5.12. Table 5.11 shows the features significance using RBF kernel. 


\begin{tabular}{|c|c|}
\hline $\begin{array}{c}\text { Feature } \\
\text { Rank }\end{array}$ & $\begin{array}{c}\text { Feature } \\
\#\end{array}$ \\
\hline 1 & 8 \\
\hline 2 & 13 \\
\hline 3 & 12 \\
\hline 4 & 15 \\
\hline 5 & 5 \\
\hline 6 & 10 \\
\hline 7 & 14 \\
\hline 8 & 4 \\
\hline 9 & 7 \\
\hline 10 & 6 \\
\hline 11 & 11 \\
\hline 12 & 3 \\
\hline 13 & 2 \\
\hline 14 & 1 \\
\hline 15 & 9 \\
\hline
\end{tabular}

Table 5.11 Macroeconomic Features Significance Ranking using Sequential Feature and RBF kernel

\begin{tabular}{|c|c|}
\hline \# of Features & Out-of-Sample Accuracy \\
\hline 1 & $55.09 \%$ \\
\hline 2 & $55.55 \%$ \\
\hline 3 & $58.33 \%$ \\
\hline 4 & $53.70 \%$ \\
\hline 5 & $54.16 \%$ \\
\hline 6 & $53.93 \%$ \\
\hline 7 & $53.70 \%$ \\
\hline 8 & $53.47 \%$ \\
\hline 9 & $53.70 \%$ \\
\hline 10 & $52.54 \%$ \\
\hline 11 & $52.31 \%$ \\
\hline 12 & $54.39 \%$ \\
\hline 13 & $56.25 \%$ \\
\hline 14 & $55.79 \%$ \\
\hline 15 & $55.79 \%$ \\
\hline
\end{tabular}

49 
Table 5.12 Sequential Feature Selection accuracy for macroeconomic data and 'zscore normalization and RBF Kernel

\subsubsection{Sequential Feature Reduction with Technical Features}

Linear Kernel:

The significance and ranking of each of the technical features can be seen in Table 5.13.

\begin{tabular}{|c|c|}
\hline $\begin{array}{c}\text { Feature } \\
\text { Rank }\end{array}$ & $\begin{array}{c}\text { Feature } \\
\#\end{array}$ \\
\hline 1 & 9 \\
\hline 2 & 15 \\
\hline 3 & 1 \\
\hline 4 & 2 \\
\hline 5 & 3 \\
\hline 6 & 6 \\
\hline 7 & 14 \\
\hline 8 & 10 \\
\hline 9 & 5 \\
\hline 10 & 7 \\
\hline 11 & 4 \\
\hline 12 & 17 \\
\hline 13 & 8 \\
\hline 14 & 16 \\
\hline 15 & 11 \\
\hline 16 & 12 \\
\hline 17 & 13 \\
\hline
\end{tabular}

Table 5.13 Sequential Feature Selection for technical data and linear kernel

Feature reduction with linear kernel and technical features showed an improvement in the out-of-sample classification that can be seen in Table 5.13. 
To achieve the best performance for the classifier, less features was given to the classifier. Using only 2 features (please refer to Table 4.2,) we were able to get $57.87 \%$ accuracy. The more features we add, the worse the performance became.

\begin{tabular}{|c|c|}
\hline $\begin{array}{c}\text { \# of } \\
\text { Features }\end{array}$ & Out-of-Sample Accuracy \\
\hline 1 & $55.78 \%$ \\
\hline 2 & $57.87 \%$ \\
\hline 3 & $56.71 \%$ \\
\hline 4 & $57.17 \%$ \\
\hline 5 & $55.32 \%$ \\
\hline 6 & $54.16 \%$ \\
\hline 7 & $53.70 \%$ \\
\hline 8 & $53.70 \%$ \\
\hline 9 & $54.63 \%$ \\
\hline 10 & $53.24 \%$ \\
\hline 11 & $55.78 \%$ \\
\hline 12 & $53.93 \%$ \\
\hline 13 & $54.63 \%$ \\
\hline 14 & $52.77 \%$ \\
\hline 15 & $52.54 \%$ \\
\hline 16 & $51.62 \%$ \\
\hline 17 & $51.15 \%$ \\
\hline
\end{tabular}

Table 5.14 Sequential Feature Selection for technical data and 'zscore' normalization and Linear Kernel

RBF:

Another test of feature reduction was performed to the technical features but this time using an RBF kernel. Using technical features with RBF kernel, the more features we added, the better the accuracy we achieved. Classifying using all features resulted in the best accuracy than classifying with a reduced number 
of features. The best accuracy achieved was $59.26 \%$. We got the second best results with only 9 features achieving an accuracy of $58.33 \%$. Table 5.15 shows the significance features using RBF kernel.

\begin{tabular}{|c|c|}
\hline $\begin{array}{c}\text { Feature } \\
\text { Rank }\end{array}$ & $\begin{array}{c}\text { Feature } \\
\#\end{array}$ \\
\hline 1 & 1 \\
\hline 2 & 14 \\
\hline 3 & 2 \\
\hline 4 & 8 \\
\hline 5 & 12 \\
\hline 6 & 7 \\
\hline 7 & 3 \\
\hline 8 & 6 \\
\hline 9 & 15 \\
\hline 10 & 17 \\
\hline 11 & 10 \\
\hline 12 & 4 \\
\hline 13 & 9 \\
\hline 14 & 5 \\
\hline 15 & 11 \\
\hline 16 & 16 \\
\hline 17 & 13 \\
\hline
\end{tabular}

Table 5.15 Sequential Feature Selection for technical data and RBF kernel

\begin{tabular}{|c|c|}
\hline $\begin{array}{c}\# \text { of } \\
\text { Features }\end{array}$ & Out-of-Sample Accuracy \\
\hline 1 & $50 \%$ \\
\hline 2 & $50.46 \%$ \\
\hline 3 & $51.85 \%$ \\
\hline 4 & $53.70 \%$ \\
\hline 5 & $54.39 \%$ \\
\hline 6 & $54.16 \%$ \\
\hline 7 & $54.63 \%$ \\
\hline 8 & $58.10 \%$ \\
\hline 9 & $58.33 \%$ \\
\hline
\end{tabular}




\begin{tabular}{|l|l|}
\hline 10 & $54.63 \%$ \\
\hline 11 & $55.09 \%$ \\
\hline 12 & $54.39 \%$ \\
\hline 13 & $55.55 \%$ \\
\hline 14 & $55.78 \%$ \\
\hline 15 & $57.63 \%$ \\
\hline 16 & $58.33 \%$ \\
\hline 17 & $59.26 \%$ \\
\hline
\end{tabular}

Table 5.16 Sequential Feature Selection for technical data and 'zscore' normalization and RBF Kernel

\subsubsection{Rankfeatures}

A simpler approach to identify the significant features was to assume all values are independent and compute a two-way t-test. Computing this test will return the index with features ranked in order of their effectiveness ranked with criterion absolute value. This test was used to relate whether the average difference between two groups was really significant or if it was due instead to data being random and not associated with each other [30]. We used the Matlab Rankfeatures function available in the bioinformatics toolbox.

Macroeconomic Features:

\begin{tabular}{|c|c|}
\hline $\begin{array}{l}\text { Feature } \\
\text { Rank }\end{array}$ & $\begin{array}{l}\text { Feature } \\
\#\end{array}$ \\
\hline 1 & 14 \\
\hline 2 & 11 \\
\hline 3 & 8 \\
\hline 4 & 9 \\
\hline 5 & 10 \\
\hline 6 & 15 \\
\hline 7 & 7 \\
\hline
\end{tabular}




\begin{tabular}{|c|c|}
\hline 8 & 4 \\
\hline 9 & 2 \\
\hline 10 & 1 \\
\hline 11 & 6 \\
\hline 12 & 13 \\
\hline 13 & 3 \\
\hline 14 & 5 \\
\hline 15 & 12 \\
\hline
\end{tabular}

Table 5.17 Macroeconomic features significance with Rankfeatures

Table 5.17 shows the features significance in order using Rankfeatures.

\begin{tabular}{|c|c|}
\hline Feature Rank & Out-of-Sample Accuracy \\
\hline 1 & $60.42 \%$ \\
\hline 2 & $53.24 \%$ \\
\hline 3 & $52.54 \%$ \\
\hline 4 & $52.31 \%$ \\
\hline 5 & $51.85 \%$ \\
\hline 6 & $53.24 \%$ \\
\hline 7 & $53.47 \%$ \\
\hline 8 & $53.24 \%$ \\
\hline 9 & $52.31 \%$ \\
\hline 10 & $53.47 \%$ \\
\hline 11 & $55.78 \%$ \\
\hline 12 & $56.02 \%$ \\
\hline 13 & $56.02 \%$ \\
\hline 14 & $56.02 \%$ \\
\hline 15 & $55.79 \%$ \\
\hline
\end{tabular}

Table 5.18 Rankfeatures Accuracy with macroeconomic features

Table 5.18 shows the accuracy of the classifier with Rankfeatures reducing the input features with RBF kernel and 'zscore' normalizing. Rankfeatures showed an improvement with the reduced features compared to using all features with 
macroeconomic by improving the out-of-sample accuracy to $60.42 \%$ using the inflation feature.

Technical Features:

Table 5.19 shows the ranking of features significance for the technical features.

\begin{tabular}{|c|c|}
\hline Ranking & Feature \\
\hline 1 & 3 \\
\hline 2 & 7 \\
\hline 3 & 9 \\
\hline 4 & 16 \\
\hline 5 & 14 \\
\hline 6 & 4 \\
\hline 7 & 5 \\
\hline 8 & 6 \\
\hline 9 & 17 \\
\hline 10 & 2 \\
\hline 11 & 10 \\
\hline 12 & 13 \\
\hline 13 & 1 \\
\hline 14 & 15 \\
\hline 15 & 8 \\
\hline 16 & 12 \\
\hline 17 & 11 \\
\hline
\end{tabular}

Table 5.19 Technical features significance with Rankfeatures

\begin{tabular}{|c|c|}
\hline$\#$ of Features & Out-of-Sample Accuracy \\
\hline 1 & $50.23 \%$ \\
\hline 2 & $55.79 \%$ \\
\hline 3 & $55.56 \%$ \\
\hline 4 & $56.48 \%$ \\
\hline 5 & $55.56 \%$ \\
\hline 6 & $55.09 \%$ \\
\hline
\end{tabular}




\begin{tabular}{|c|c|}
\hline 7 & $54.86 \%$ \\
\hline 8 & $56.02 \%$ \\
\hline 9 & $54.86 \%$ \\
\hline 10 & $52.55 \%$ \\
\hline 11 & $54.17 \%$ \\
\hline 12 & $55.79 \%$ \\
\hline 13 & $54.86 \%$ \\
\hline 14 & $56.25 \%$ \\
\hline 15 & $56.48 \%$ \\
\hline 16 & $55.32 \%$ \\
\hline 17 & $59.26 \%$ \\
\hline
\end{tabular}

Table 5.20 Rankfeatures Accuracy with technical features

Table 5.20 shows how technical features did not respond well to

Rankfeatures. Rankfeatures did not identify the significant features in comparison with Sequential Feature Selection for the technical features. Most of the out-of-sample accuracies were not close to the best classification result when including all features. Overall, Rankfeatures worked better with macroeconomic features compared to technical features.

\subsection{Combining Macroeconomic with Technical Features}

One of the conclusions in the paper "A Comparison of PNN and SVM for Stock Market Trend Prediction using Economic and Technical Information," was that predicting the market using macroeconomic data was more accurate when compared to technical indicators [31]. It also contends that the addition of both macroeconomic and technical features does not improve the classification accuracy. After running the set of technical features and feature reduction, we found that the best classification accuracy is achieved when all features are 
used. Since the more features we add to the RBF classifier, we decided to expand the initial set of features by combining both the macroeconomic and technical features. The next step of this work after combining macroeconomic and technical features was to do a feature reduction to find the best classification accuracy. When combining all macroeconomic and technical features together, we were able to obtain $59.72 \%$ classification accuracy. This is only marginally better than using technical features alone.

\begin{tabular}{|c|c|c|c|c|}
\hline Procedure & $\begin{array}{c}\text { Out-of-Sample } \\
\text { Accuracy }\end{array}$ & \# of Features & C & Sigma \\
\hline $\begin{array}{c}\text { Combining Macroeconomic } \\
\text { with Technical Features }\end{array}$ & $59.72 \%$ & 32 & 1 & 1 \\
\hline
\end{tabular}

Table 5.21 Combination of macroeconomic and technical features

\subsection{Summary of Results}

Tables 5.22 and 5.23 are a summary of the best performing results and show a comparison between the experiments using macroeconomic and technical features alone. Improved accuracies were further obtained optimizing the SVM RBF parameters. The optimized parameter values are shown in the tables. 


\begin{tabular}{|c|c|c|c|c|}
\hline Procedure & $\begin{array}{c}\text { Out-of-Sample } \\
\text { Accuracy }\end{array}$ & \# of Features & C & Sigma \\
\hline All features & $56.48 \%$ & 15 & 1 & 1 \\
\hline Sequential Feature Reduction & $60.41 \%$ & 1 & 1 & $\begin{array}{c}\text { NA } \\
\text { (linear) }\end{array}$ \\
\hline Rankfeatures & $60.42 \%$ & 1 & 1 & 1 \\
\hline RBF Parameters Optimization & $59.25 \%$ & 15 & 1 & 0.45 \\
\hline
\end{tabular}

Table 5.22 Summary of the performance results using macroeconomic features

\begin{tabular}{|c|c|c|c|c|}
\hline Procedure & $\begin{array}{c}\text { Out-of-Sample } \\
\text { Accuracy }\end{array}$ & \# of Features & C & Sigma \\
\hline All features & $59.26 \%$ & 17 & 1 & 1 \\
\hline Sequential Feature Reduction & $58.33 \%$ & 9 & 1 & 1 \\
\hline Rankfeatures & $56.48 \%$ & 4 & 1 & 1 \\
\hline RBF Parameters Optimization & $62.04 \%$ & 17 & 1 & 0.425 \\
\hline
\end{tabular}

Table 5.23 Summary of the performance results using technical features

From the above results, we can conclude the overall performance of the technical features was better than macroeconomic features in contrast to the result given in [31].

\subsection{Comparison between Predictions Based on Basic Assumptions and SVM}

The overall trend of the market over the long term is in the up direction. The next step was to evaluate and compare the classifier prediction performance during 3 different periods. The first period was the overall out-ofsample period from January 1975 - December 2010. The two other periods were when the US economy was hit by financial crisis. The first financial crisis period considered was from October 2000 - September 2002. This period represents the period of the bursting of the dot com bubble. The second period 
was from October 2007 - July 2009, which was during the credit crisis. The classification was performed using zscore, RBF kernel and technical features. The first procedure was a comparison of the prediction which assumes a monthly trend of 'up' for all months considered during the out-of-sample period. Then we did the opposite by comparing the prediction results with down only during the out-of-sample period. The next procedure was to compare the results with a naïve prediction where if the previous month was up (or down), then the next month is predicted to be up (or down). The last procedure was to do the reverse of the previous procedure where if the previous month was up (or down), then the next month is predicted to be down (or up).

\begin{tabular}{|c|c|}
\hline Procedure & Out-Of-Sample Accuracy $\%$ \\
\hline Up Only & $60.42 \%$ \\
\hline Down Only & $39.58 \%$ \\
\hline Following Previous Month & $52.55 \%$ \\
\hline Opposite of Previous Month & $47.22 \%$ \\
\hline SVM Prediction & $62.04 \%$ \\
\hline
\end{tabular}

Table 5.24 Results for comparing the classification accuracy during the full out-of-sample period

We can see from Table 5.24 that when assuming the index will go up every month, the overall accuracy was $60.4 \%$. That was true for most of the time prior to 2000. After that period, the index direction wasn't going up only but rather oscillated.

The next step was to compare the results during the first period when the market crashed. Figure 5.3 shows the index price during the first economic crisis we were looking at. 


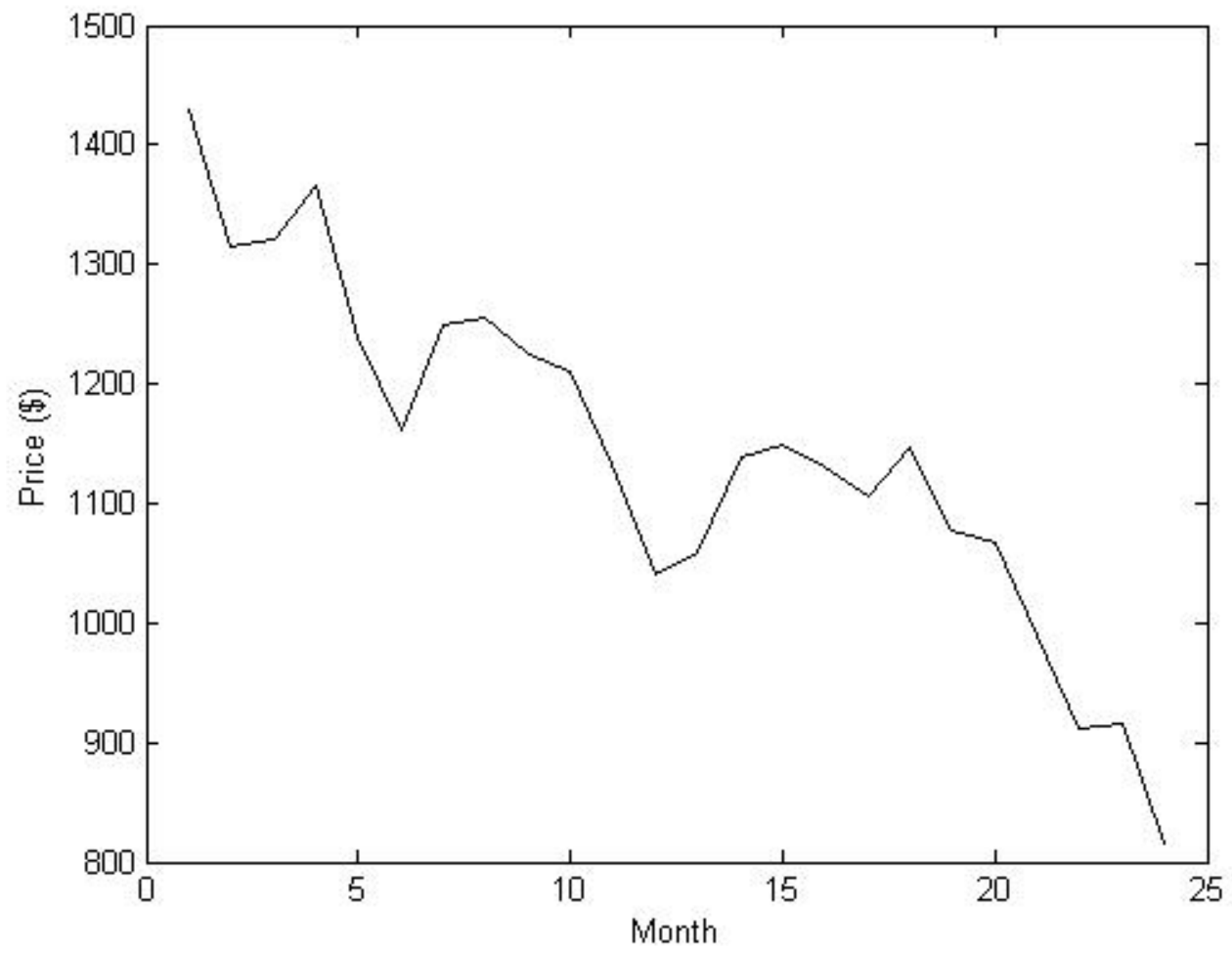

Figure 5.3 S\&P 500 Index price over the first economic crisis (October 2000 - September 2002)

The results of the predictions are provided below:

\begin{tabular}{|c|c|}
\hline Procedure & Out-Of-Sample Accuracy \% \\
\hline Up Only & $41.67 \%$ \\
\hline Down Only & $58.33 \%$ \\
\hline Following Previous Month & $54.17 \%$ \\
\hline Opposite of Previous Month & $50.00 \%$ \\
\hline SVM Prediction & $50.00 \%$ \\
\hline
\end{tabular}

Table 5.25 Results for comparing the classification accuracy during the first economic crisis period

From Table 5.25, we can notice if we were to go up only from the beginning of out-of-sample, a big risk was avoided using SVM for prediction 
during this economic crisis period if the strategy were to be followed by if the index price will only go up.

The last step was to see the performance during the last economic crisis beginning in late 2007. It was clear from Figure 5.4 that the index was going down during this period.

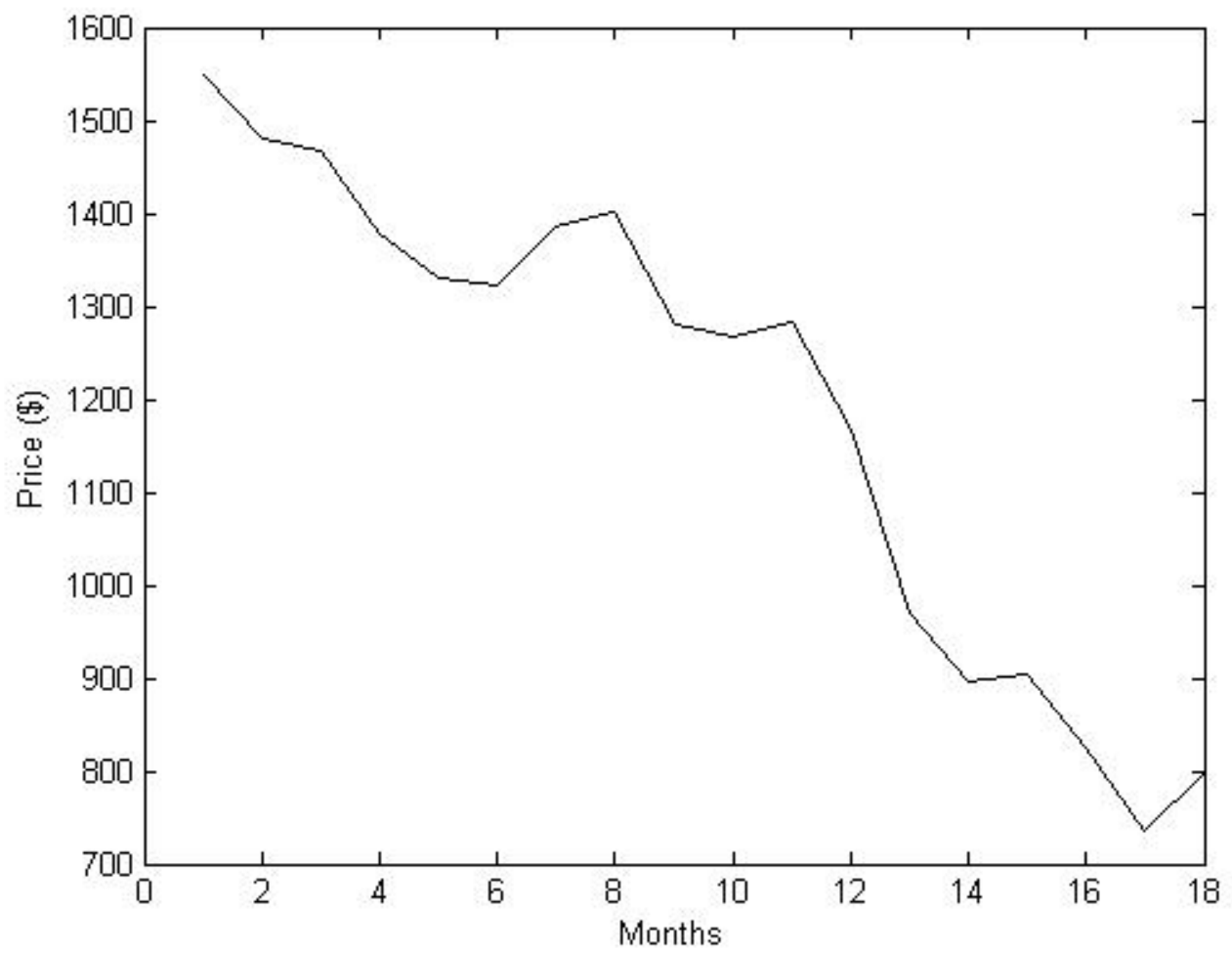

Figure 5.4 S\&P 500 Index price during the last economic crisis (October 2007 - July 2009) 


\begin{tabular}{|c|c|}
\hline Procedure & Out-Of-Sample Accuracy $\%$ \\
\hline Up Only & $33.33 \%$ \\
\hline Down Only & $66.67 \%$ \\
\hline Following Previous Month & $61.11 \%$ \\
\hline Opposite of Previous Month & $44.44 \%$ \\
\hline SVM Prediction & $61.11 \%$ \\
\hline
\end{tabular}

Table 5.26 Results for comparing the classification accuracy during the last economic crisis period

We see from Table 5.26 that if we were to use the up only prediction, then the accuracy was $33.33 \%$. SVM predicted $61.11 \%$ accurately, which as it happens was the same result when following the direction of previous month. This was another test to show the advantage of SVM over if we assumed the market will always go up.

If an investing strategy was implemented during the last two economic crisis period assuming the market will always go up, it would have encountered huge losses. The SVM reduced the risk in the first economic crisis to $50 \%$ compared to $58.33 \%$ loss when assuming the market will always go up. In the second economic crisis, SVM gave $61.1 \%$ accuracy while an up only strategy only had $33.33 \%$ accuracy. 
Chapter 6: Conclusion and Future Work

\subsection{Conclusion}

This worked performed several prediction models using SVM as a classifying tool in attempt to predict the direction of the market's trend using S\&P 500 into 'up' or 'down' months. The first model used 15 inputs for macroeconomic features and 17 for technical features, as inputs to the SVM classifier. Results showed the best accuracy of $56.48 \%$ using all macroeconomic features with zscore to normalize the data, $51.39 \%$ using normc, and $53.94 \%$ using normalize. Classification using technical features showed better results compared to macroeconomic. We were able to obtain the best classification accuracy using all technical features with zscore. With normalizing the data using zscore, we were able to get $59.26 \%$ classification accuracy, $49.77 \%$ using normc, and $53.94 \%$ using normalize.

Optimizing the RBF's parameters further and raised the out-of-sample accuracy from $59.26 \%$ to $62.04 \%$ using technical features and from $56.48 \%$ to $59.25 \%$ using macroeconomic features. However, the best result for macroeconomic data was achieved using only the inflation feature giving $60.42 \%$.

Reducing the dimensionality of the input features using Rankfeatures was found to be better in terms of choosing the right features needed and the accuracy to which features to exclude based on the RBF kernel we tested. The 
response for reducing the features was better for the macroeconomic data. The linear kernel performs better with fewer features used to classify when using Sequential Feature Selection. We were able to get $60 \%$ accuracy with only one feature using macroeconomic data.

From this work, we can conclude that classification using technical features result in better classification accuracy than using macroeconomic features. This may be fortuitous as obtaining the macroeconomic data is generally not an easy task. In contrast, technical features can be easily obtained and since all you need is the index's price to be able to derive the features used in this work.

This work was initially motivated by the result presented by Yuan in [24], where an out-of-sample classification accuracy of $86 \%$ was achieved. By the work done in the thesis we believe this rate of accuracy was achieved unfortunately by error. Specifically when labeling the correct target, Yuan's result is shifted by one month which results in looking into the future. When labeling the targets in the same way as [24], our classification rate achieved was also $86 \%$ when using technical features and close to $98 \%$ with macroeconomic features.

In the future, the parameters of the technical features can be optimized, perhaps using Genetic Algorithm to find better performing features. Also, SVM regression can be used to get a sense of what range the index can be expected to move during prediction period. 


\section{References}

[1] L. Blume and S. Durlauf, The New Palgrave: A Dictionary of Economics, Second Edition, 2007. New York: Palgrave McMillan.

[2] Kasimati, Evangelia. Macroeconomic and Financial Analysis of Mega-Events. Thesis. University of Bath, United Kingdom, 2006.

[3] Colby, Robert W. The Encyclopedia Of Technical Market Indicators. 2nd ed. Toronto: Mc Graw-Hill, 2003.

[4] Sutter, Christian, Zingg, Pascal, and Bertschi, Rolf. Credit Suisse, "Technical Analysis - Explained." Accessed August 16, 2013.

[5] Ng, Andrew. Stanford University, "Support Vector Machines." Accessed August 2, 2013.

[6] R. Sullivan, A. Timmermann, and H. White. Data-snooping, technical trading rule performance and the bootstrap. Journal of Finance, 54:1647 - 1691, 1999.

[7] Lento, C., N. Gradojevic, and C. S. Wright. "Investment Information Content in Bollinger Bands." Applied Financial Economics Letters 3.4 (2007): 263-67. Print.

[8] Martiny, Karsten. An Investigation of Machine-Learning Approaches for a Technical Analysis of Financial Markets. Thesis. Technische Universität Hamburg-Harburg, 2010.

[9] Zhang, Yingjian. Preidiction of Financial Time Serie with Hidden Markov Models. Thesis. Shandong University, China, 2001.

[10] MQL Community - Technical Analysis, "Momentum." Accessed September 20, 2013. http://ta.mql4.com/indicators/oscillators/momentum 
[11] OpenCV, "Introduction to Support Vector Machines." Accessed August 10, 2013.

http://docs.opencv.org/doc/tutorials/ml/introduction_to_svm/introduction_to_svm. html

[12] Bottou, L., and Chih-Jen Lin. National Taiwan University, Department of Computer Science, "Support Vector Machine Solvers." (2009).

[13] Weston, J. NEC Labs America, "Support Vector Machine (and Statistical Learning Theory) Tutorial." Last modified February 26, 2002.

[14] Girma, Henok. Center of Experimental Mechanics. University of Ljubljana. "A Tutorial on Support Vector Machine." (2009)

[15] Weisstein, Eric W. "Normalized Vector." From MathWorld--A Wolfram Web Resource. http://mathworld.wolfram.com/NormalizedVector.html

[16] Bergstra, James, and Bengio, Yoshua (2012). "Random Search for HyperParameter Optimization". J. Machine Learning Research 13: 281-305.

[17] Chin-Wei Hsu, Chih-Chung Chang and Chih-Jen Lin. A practical guide to support vector classification. Technical Report, National Taiwan University. (2010).

[18] Goyal, A., and Welch, I. "A comprehensive look at the empirical performance of equity premium performance." Review of Financial Studies 21. (2008). pp 1455-1508.

[19] TicTacTec, "TA-Lib : Technical Analysis Library." Accessed October 11, 2012. http://www.ta-lib.org/index.html.

[20] Vision, Learning and Robotics, "Feature Normalization for Learning Classifier." Accessed September 4, 2013. 
[21] Amibroker, "Walk-forward Testing." Accessed October 1, 2013.

[22] Fan, J. Fan, Y. and Wu, Y. Princeton University and Harvard University, "High-Dimensional Classication." Accessed October 9, 2013.

[23] Scholkopf, B., and Smola, A.J., Learning with Kernels, MIT Press, Cambridge, MA. 2002.

[24] Yuan, Charles, Washington University in St. Louis, "Predicting S\&P 500 Returns Using Support Vector Machines: Theory and Empirics." October 2011.

[25] Teixeira, Lamartine Almeida, and Adriano Lorena Oliveira. "A method for automatic stock trading combining technical analysis and nearest neighbor classification." Expert Systems with Application. Vol 37. Oct (2010): 6885-6890.

[26] Vlachos, Andreas. "Active Learning with Support Vector Machines." Thesis. University of Edinburgh, 2004.

[27] Scikit-learn Developers, "Grid Search: Searching for Estimator Parameters." http://scikit-learn.org/stable/modules/grid_search.html. Accessed 05 Oct. 2013.

[28] Joachims, Thorsten. Cornell University, "Support Vector and Kernel Methods." Accessed September 2, 2013.

[29] Hauskrecht, Milos. University of Pittsburgh, "Dimensionality Reduction Feature Selection." Accessed August 19, 2013.

[30] Weisstein, Eric W. "Paired t-Test." From MathWorld--A Wolfram Web Resource. Accessed August 11, 2013 http://mathworld.wolfram.com/PairedtTest.html

[31] Lahmiri, Salim. "A Comparison of PNN and SVM for Stock Market Trend Prediction using Economic and Technical Information." International Journal of Computer Applications (0975 - 8887). Vol 29, No 3 Sept (2011) 
[32] Kantavat, P., and Kijsirikul, B., Chulalongkorn University, "Combining

Technical Analysis and Support Vector Machine for Stock Trading." 2008 IEEE International Conference on Computational Intelligence for Financial Engineering. 


\section{Appendix: Matlab Code}

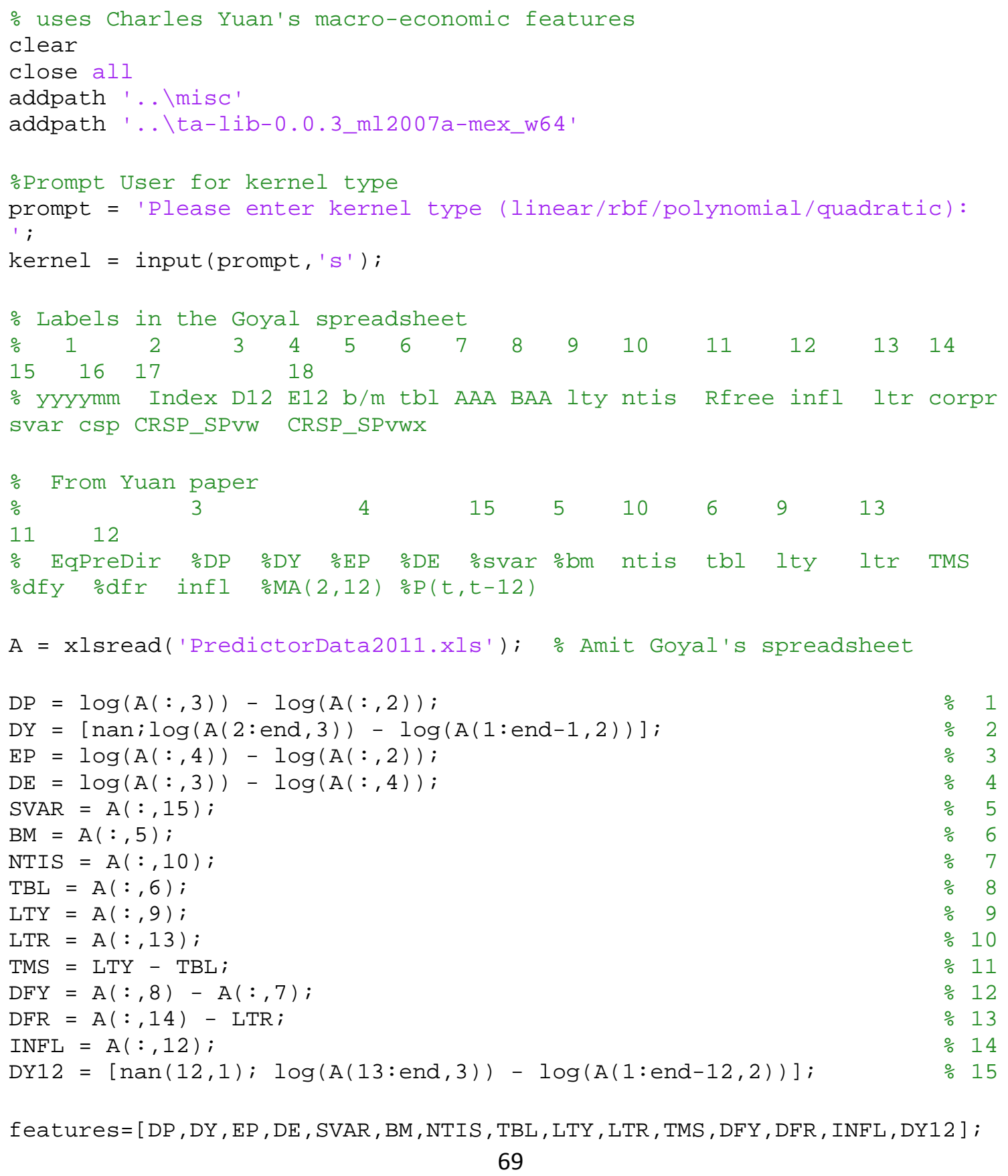

features $=[D P, D Y, E P, D E$, SVAR, BM, NTIS, TBL, LTY, LTR, TMS, DFY, DFR, INFL, DY12] ; 


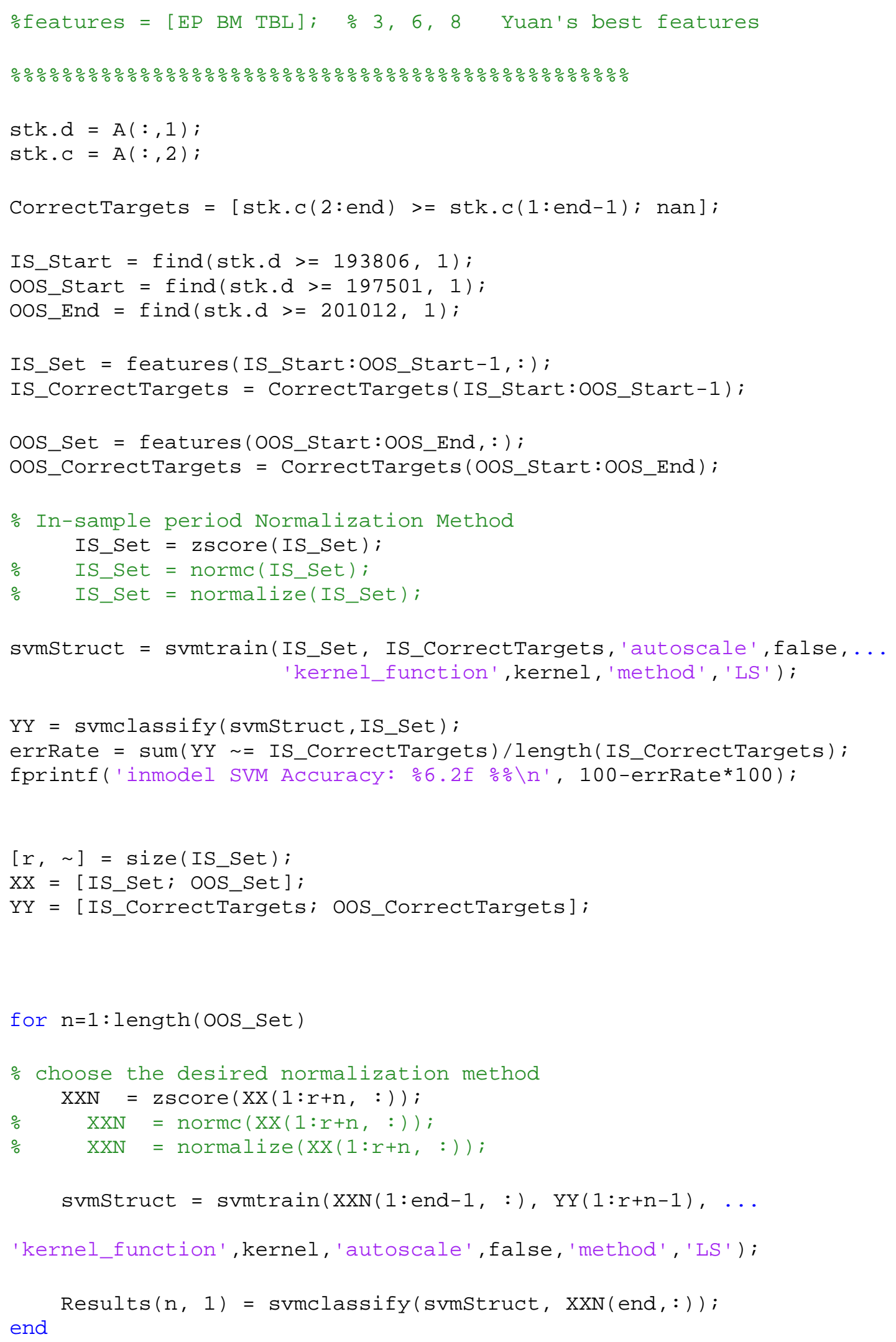




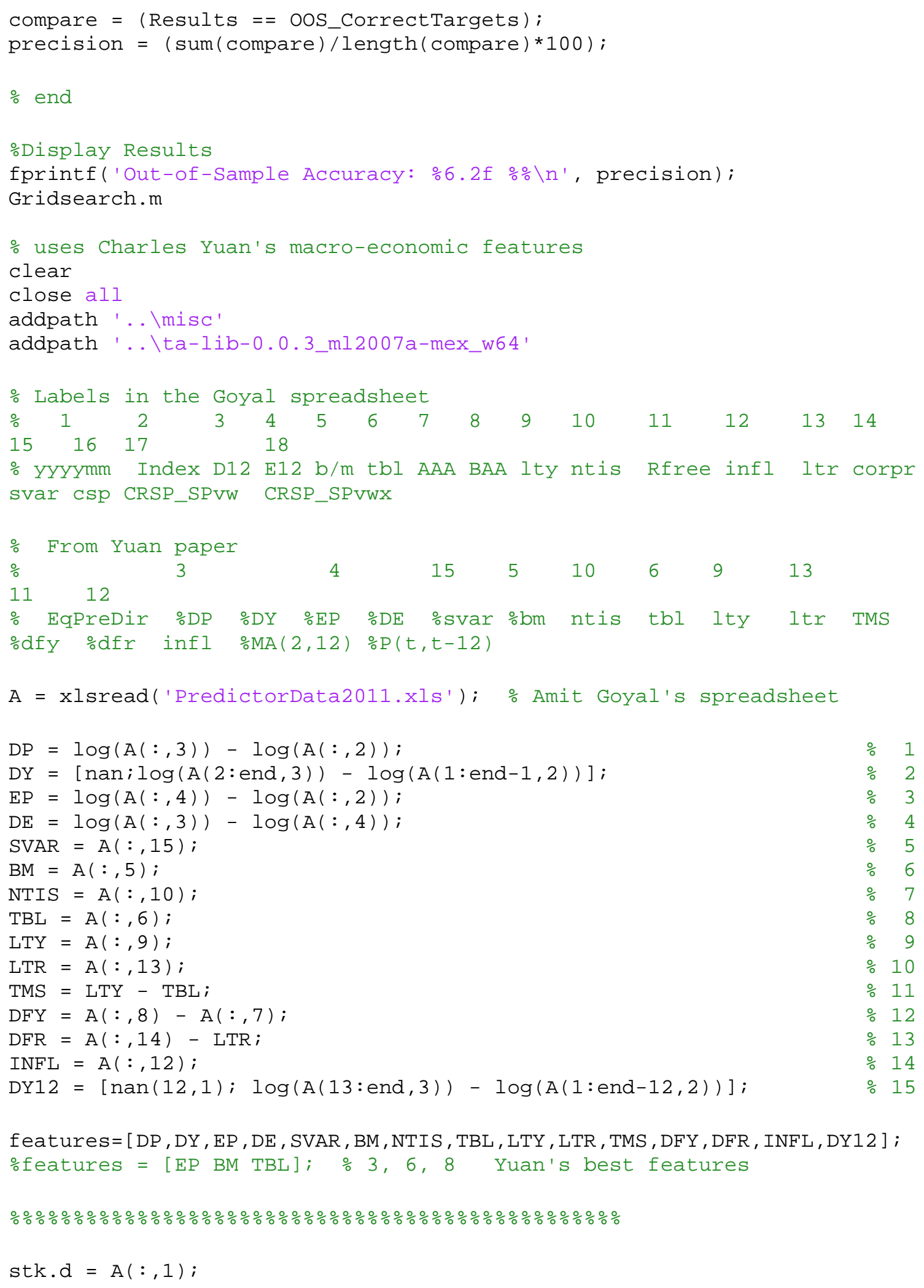




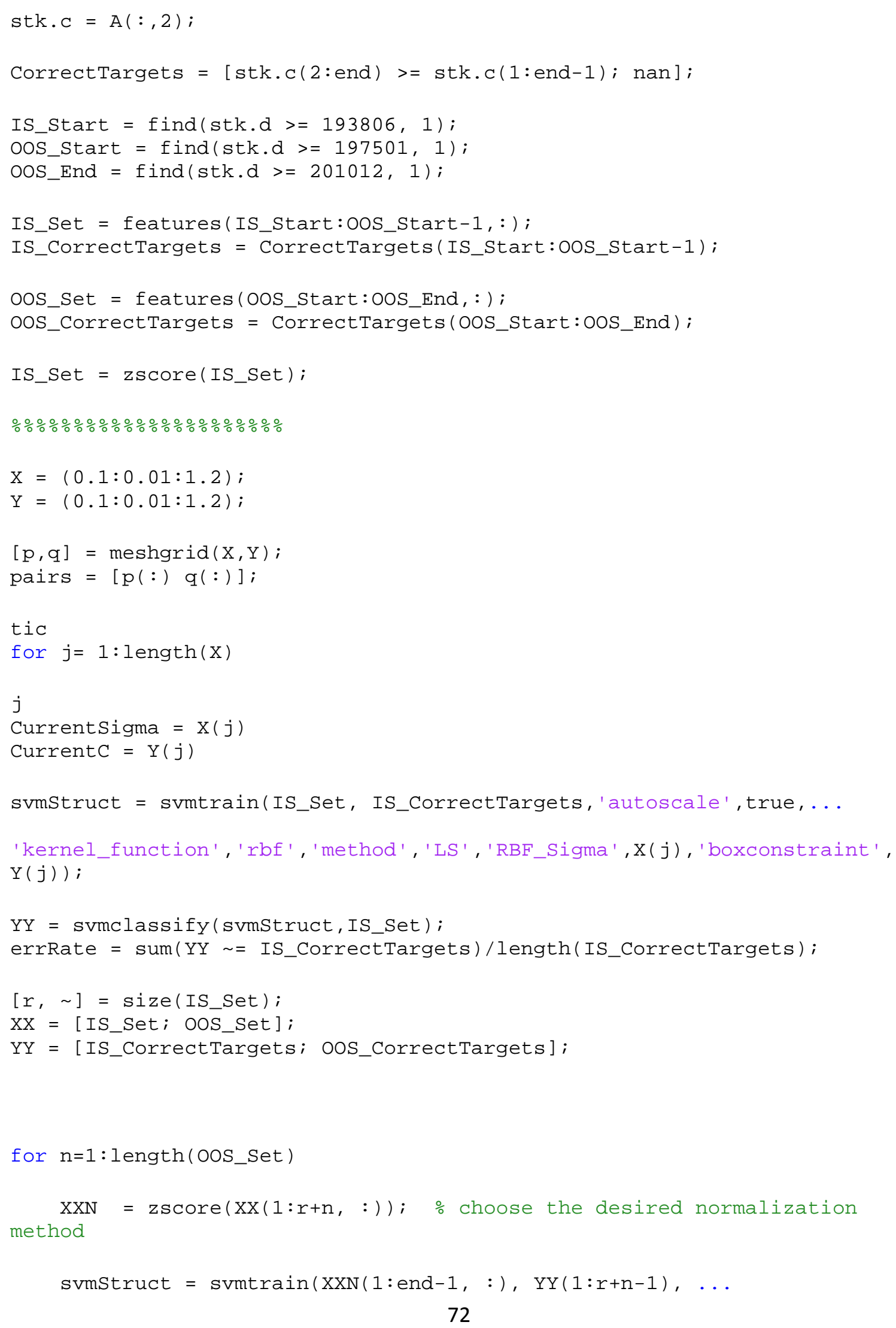




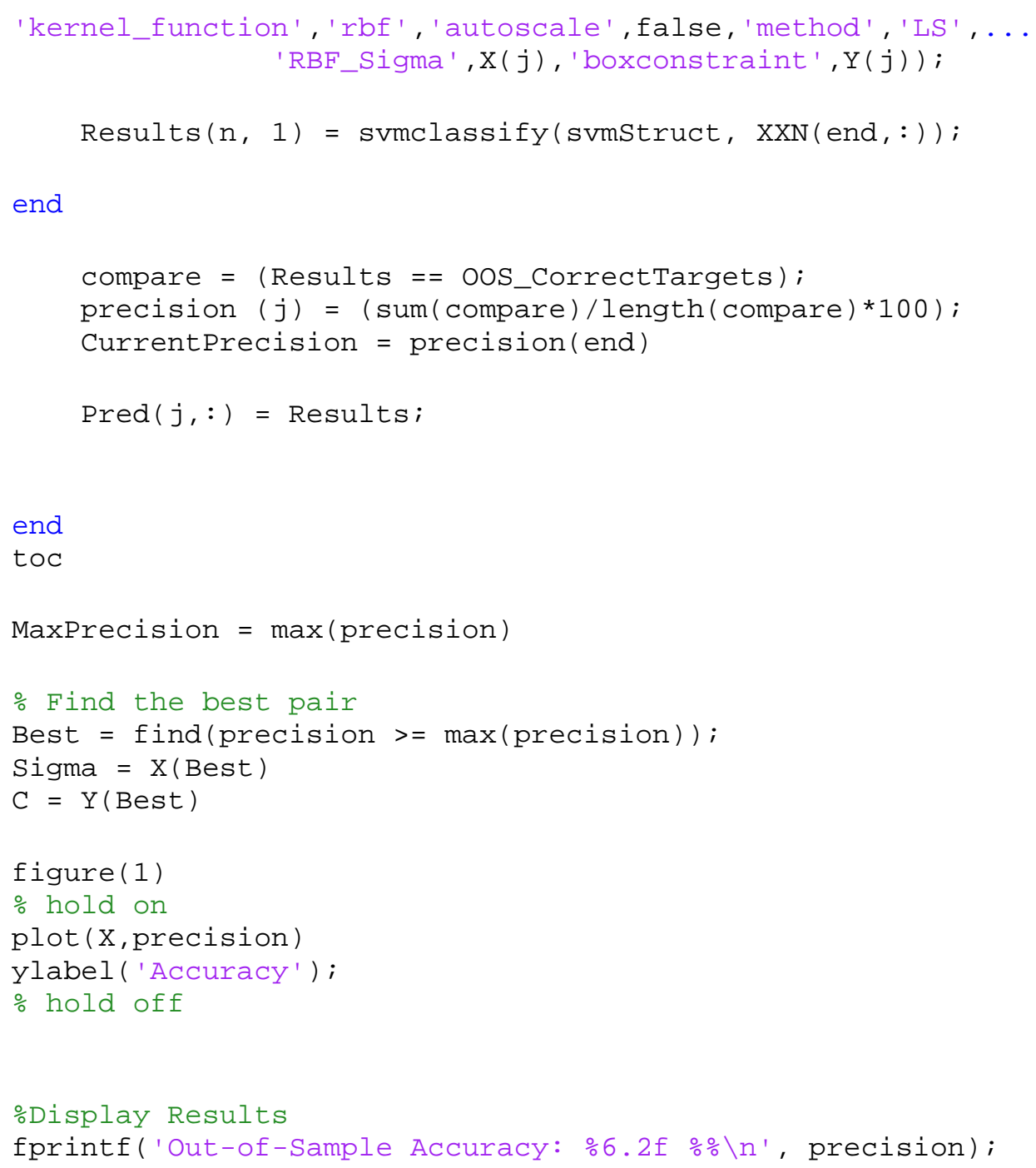




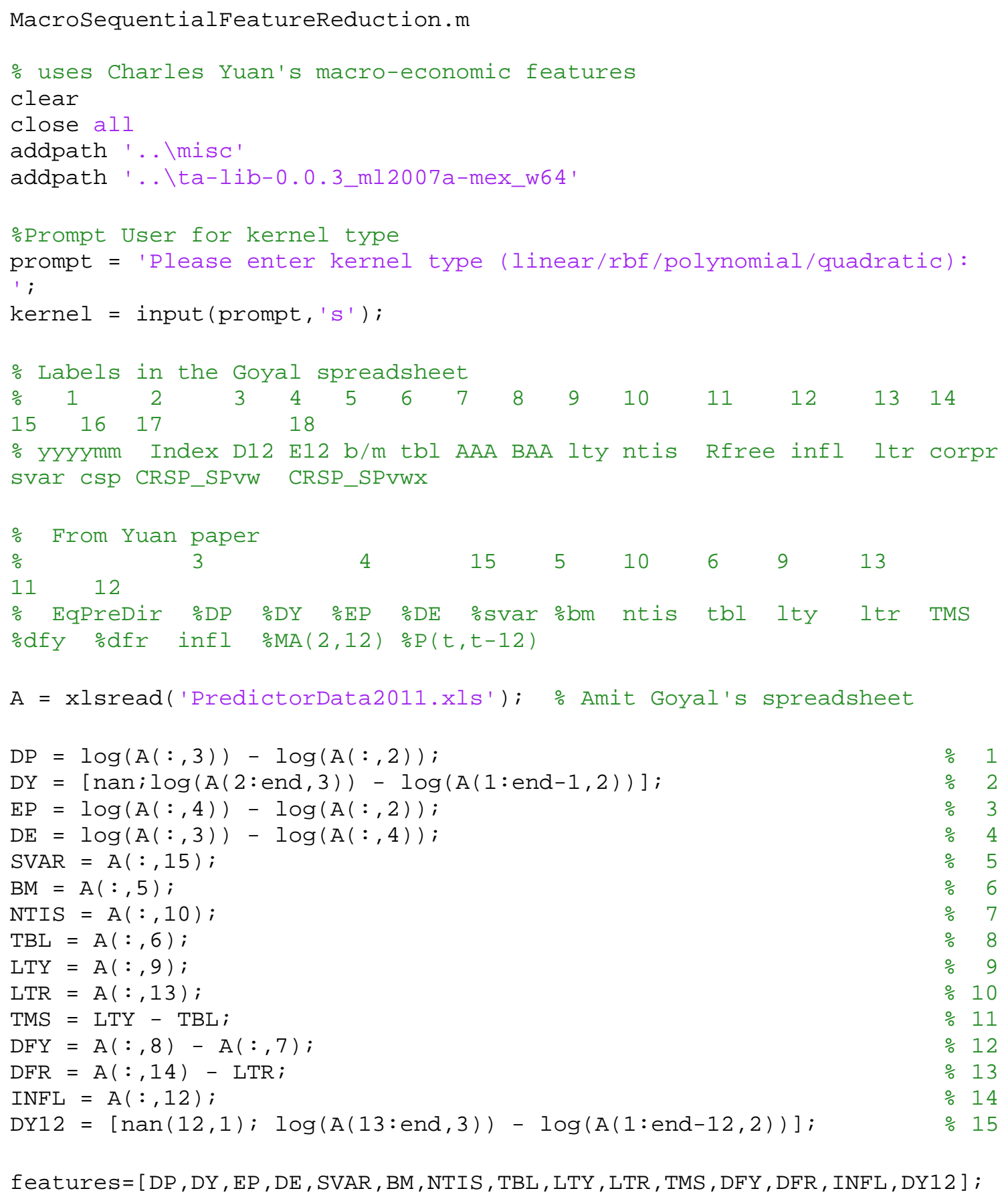




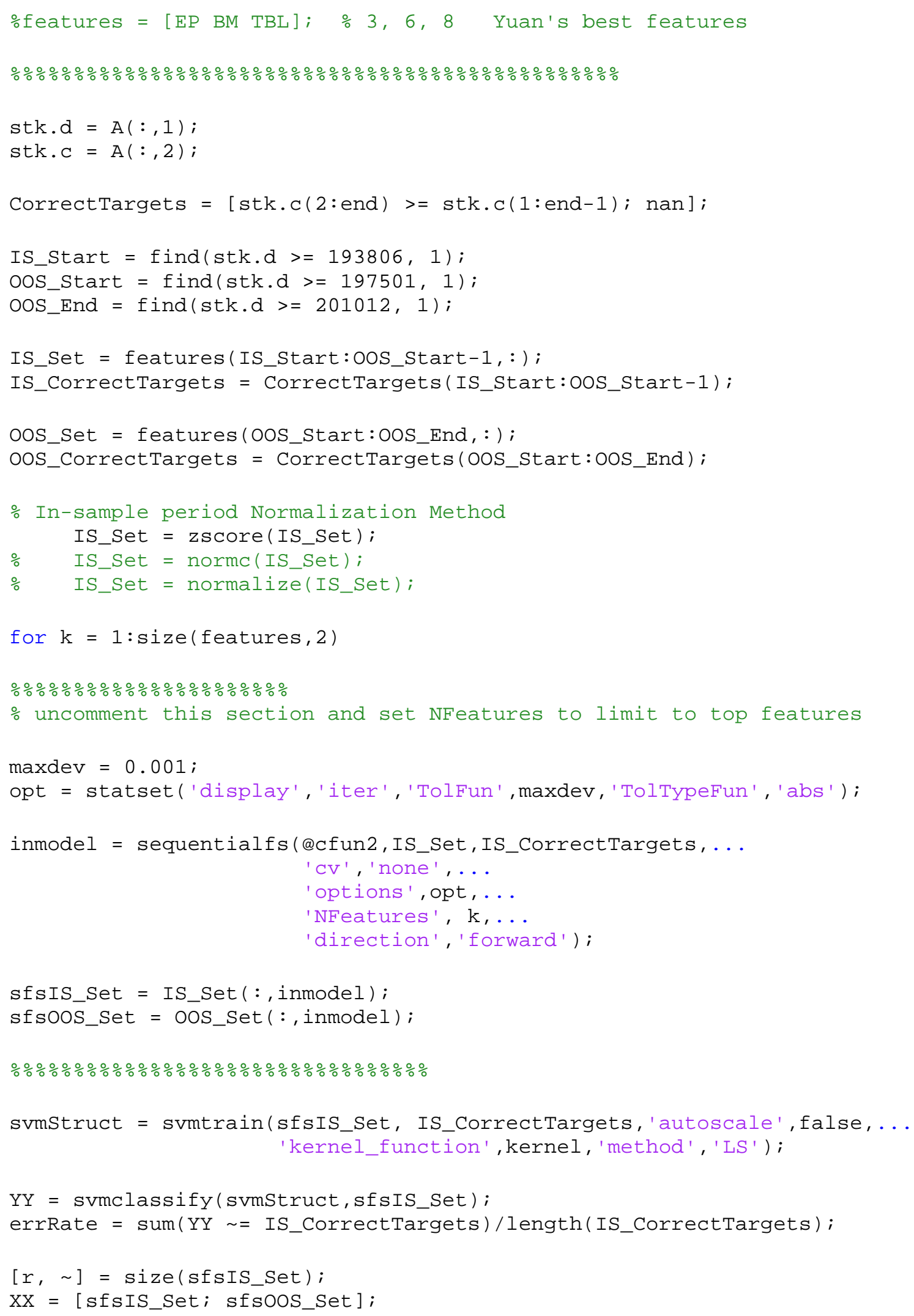




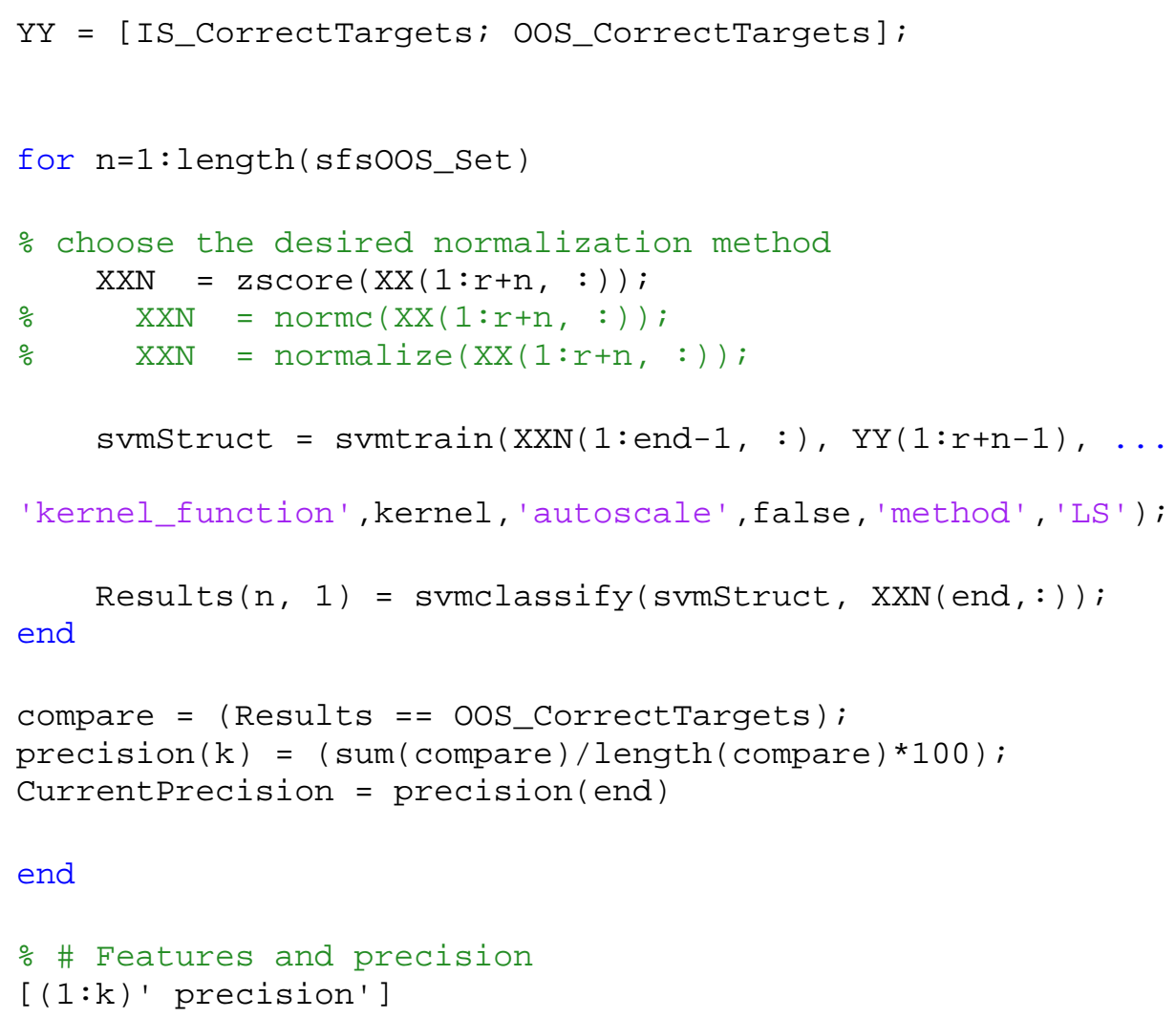




\section{Macrorankfeatures.m}

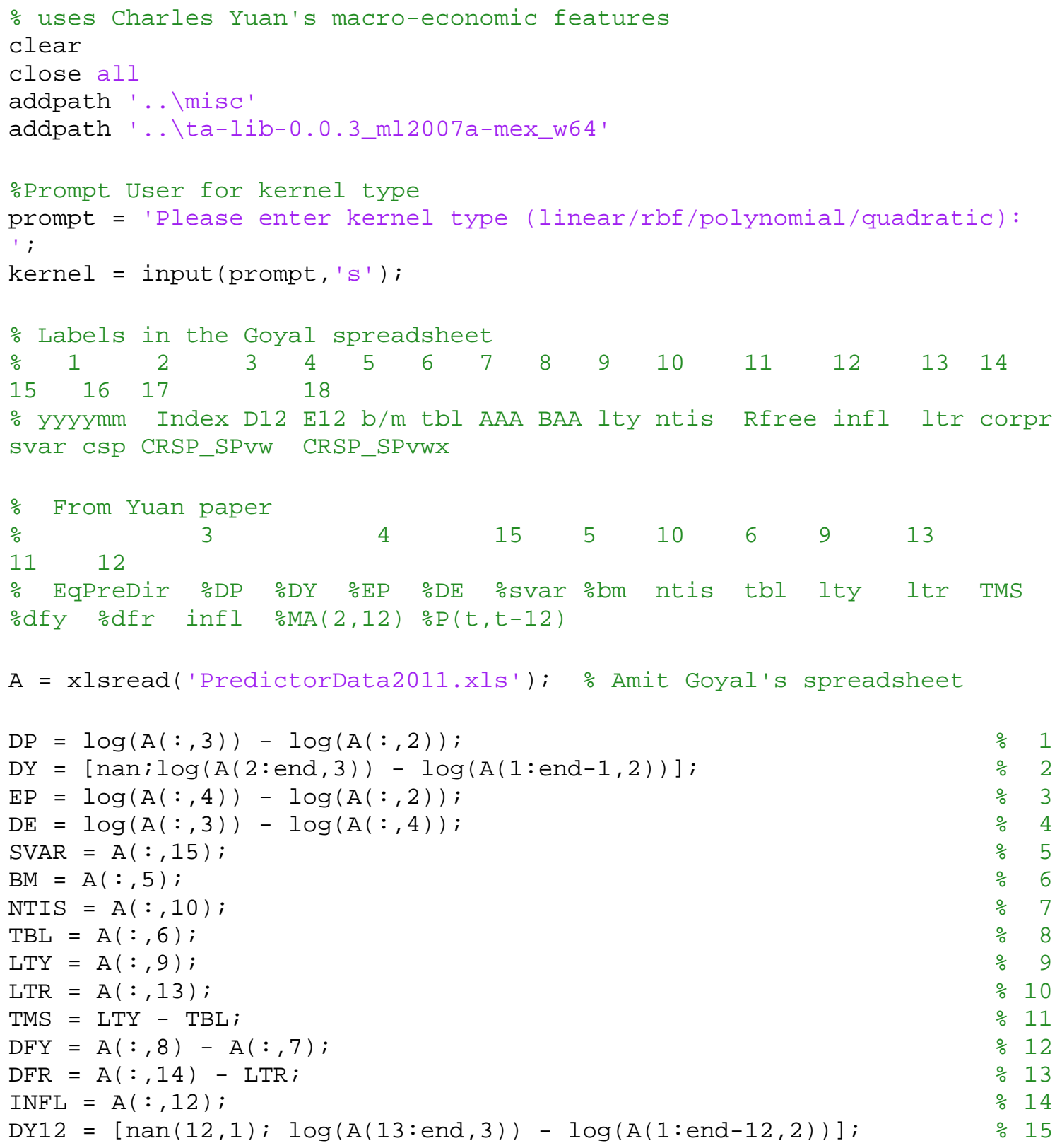

features $=[D P, D Y, E P, D E$, SVAR, BM , NTIS , TBL , LTY , LTR, TMS, DFY , DFR, INFL, DY12] ; $\%$ features $=[\mathrm{EP}$ BM TBL] $; \% 3,6,8$ Yuan's best features

\%\%\%\%\%\%\%\%\%\%\%\%\%\%\%\%\%\%\%\%\%\%\%\%\%\%\%\%\%\%\%\%\%\%\%\%\%\%\%\%\%\%

stk.d = A(:, 1);

stk. $c=A(:, 2)$;

CorrectTargets $=[$ stk.c $(2:$ end $)>=$ stk.c $(1:$ end -1$) ;$ nan $]$; 


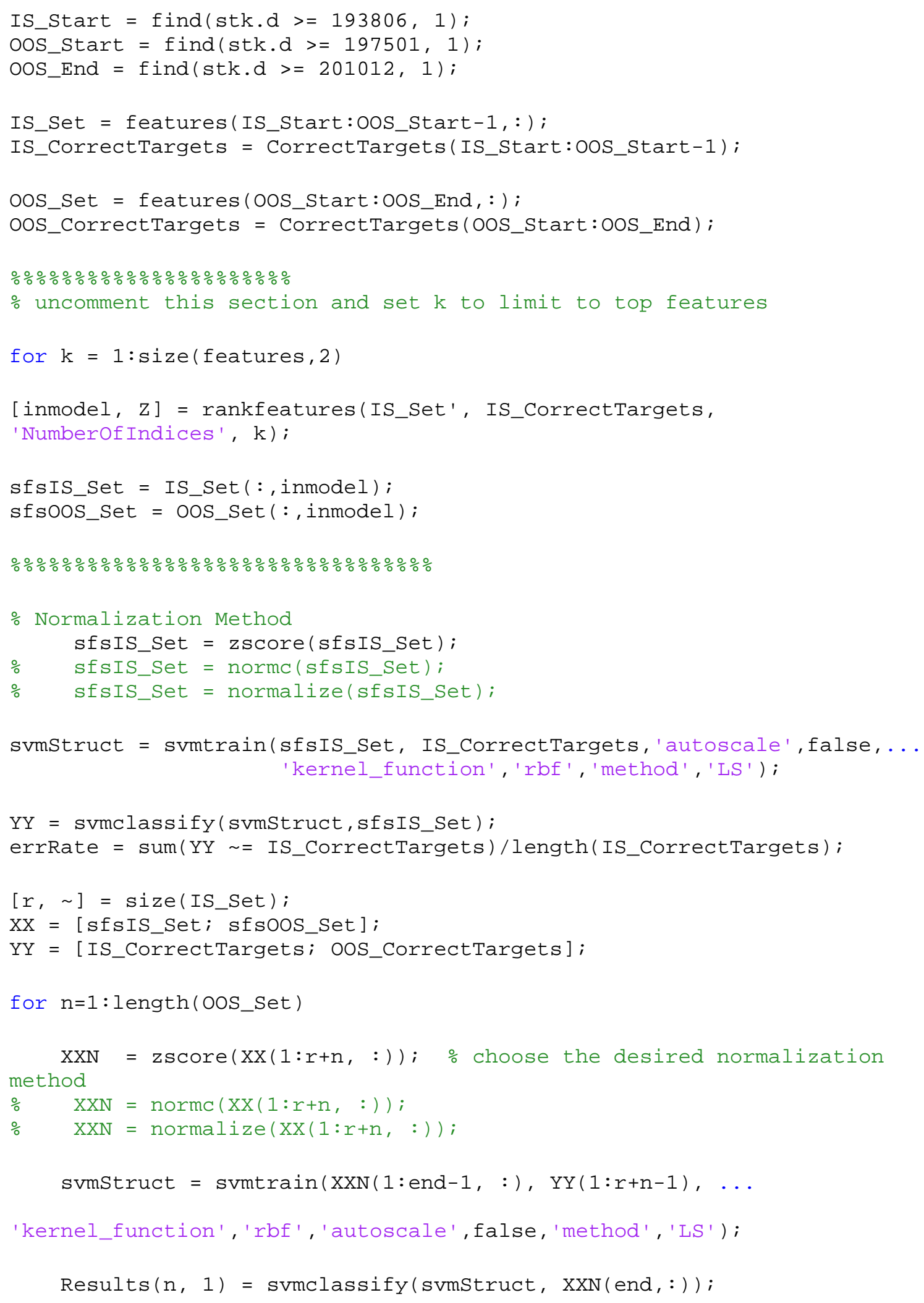




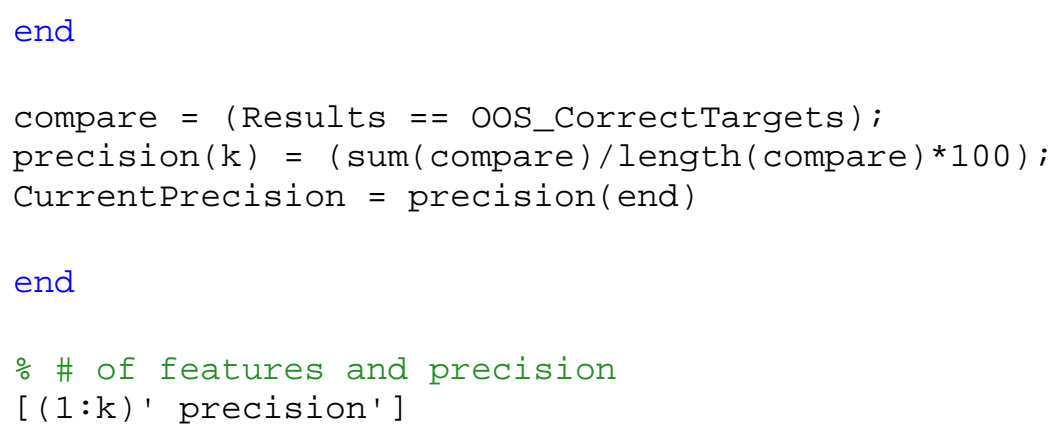




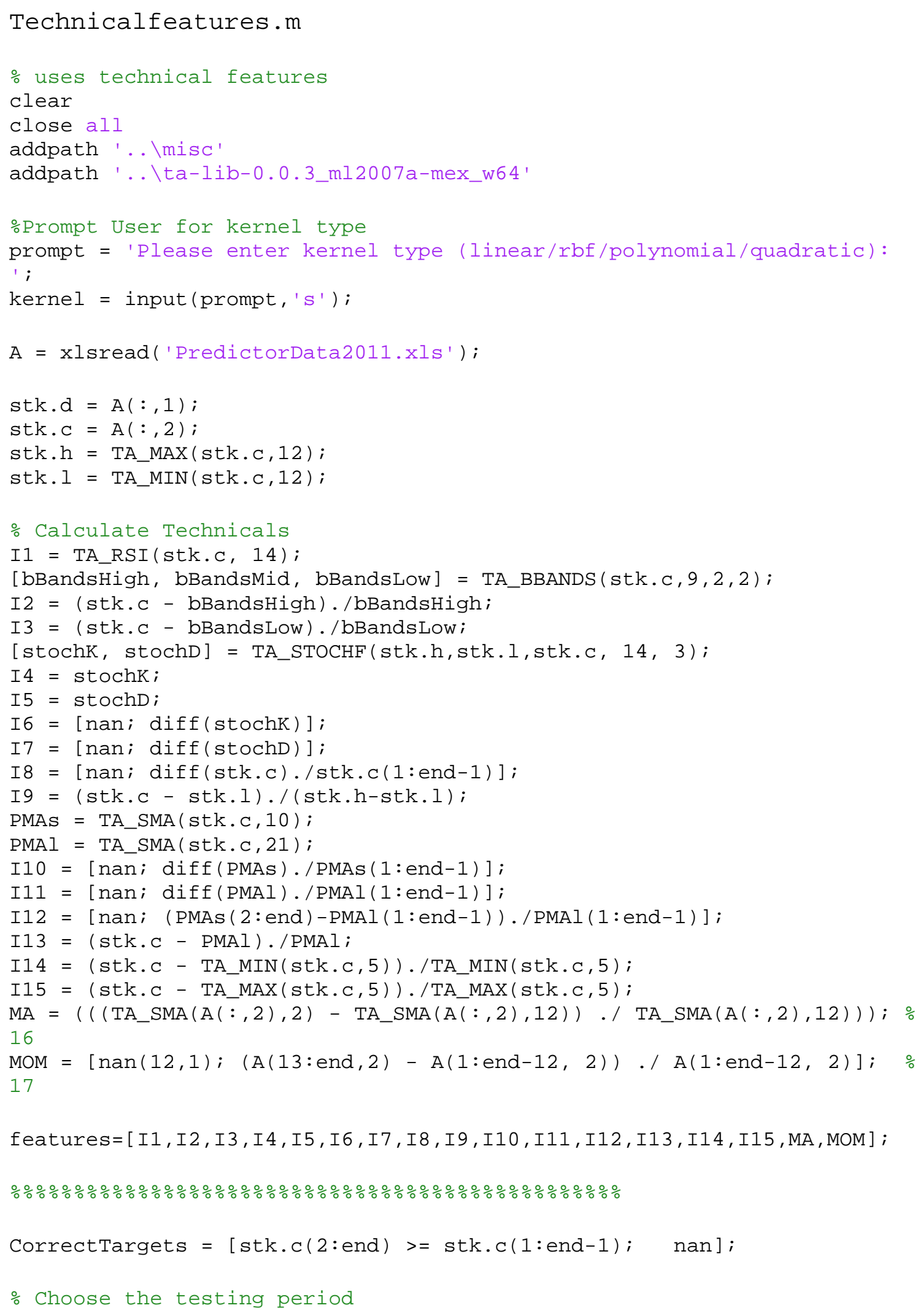




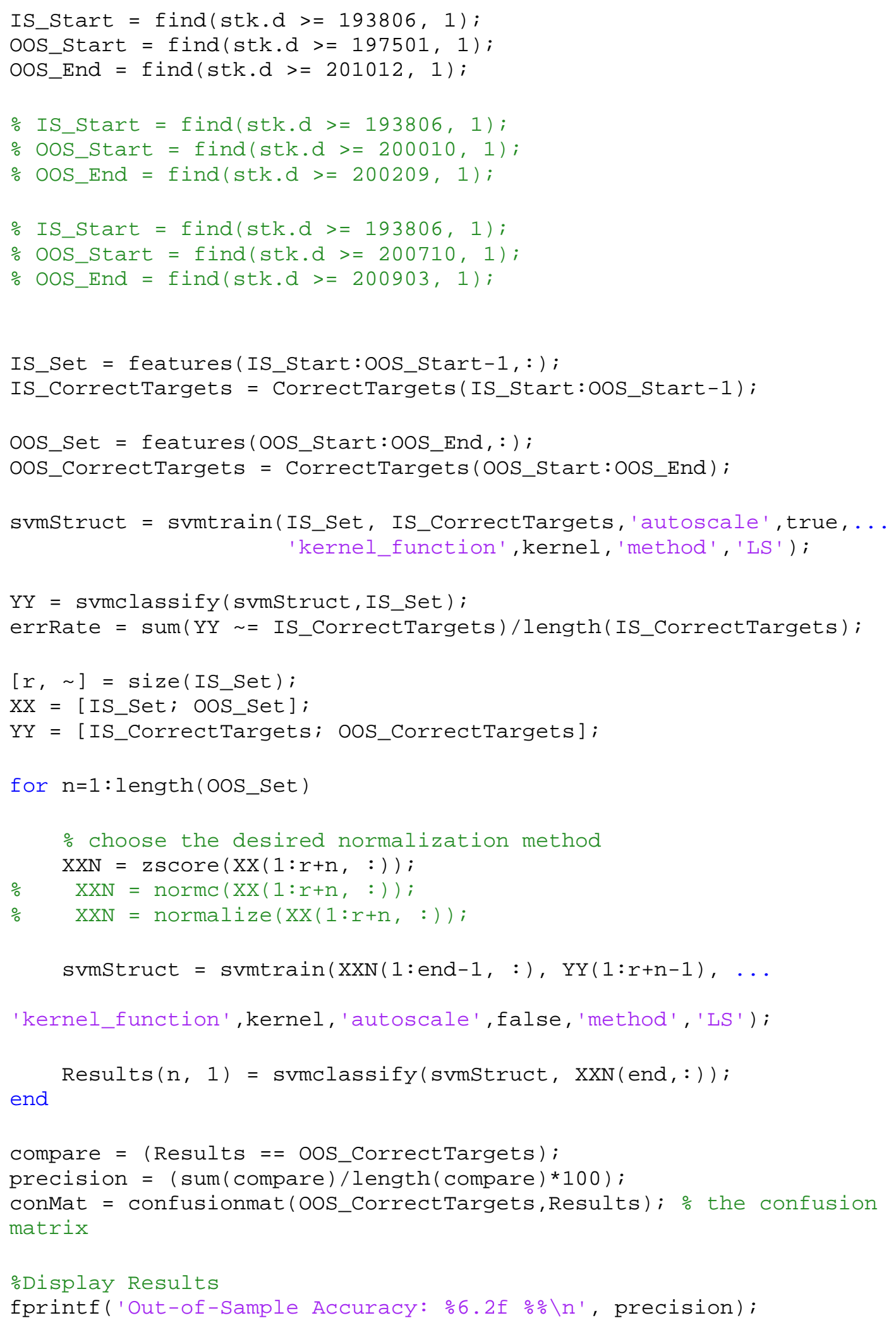




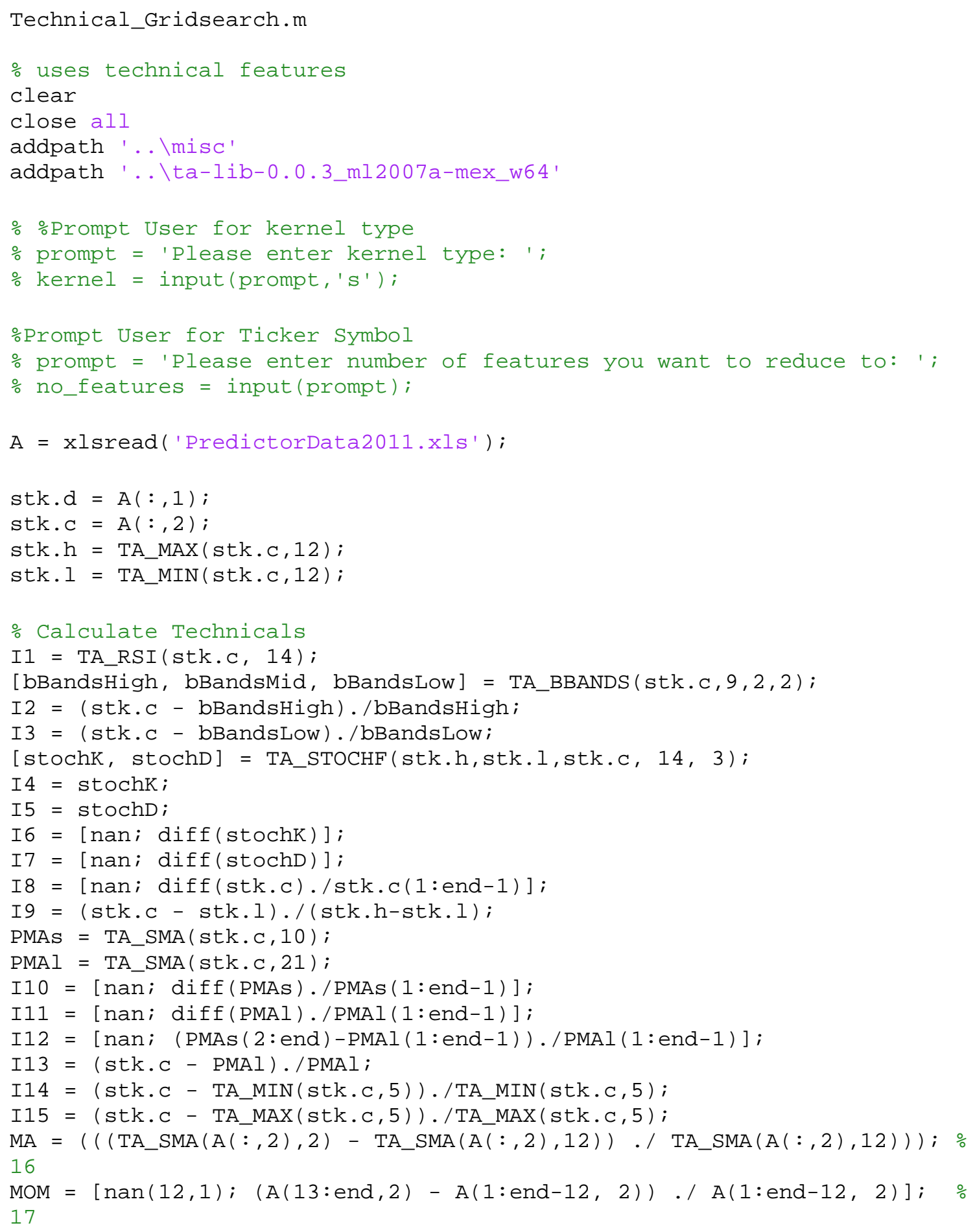




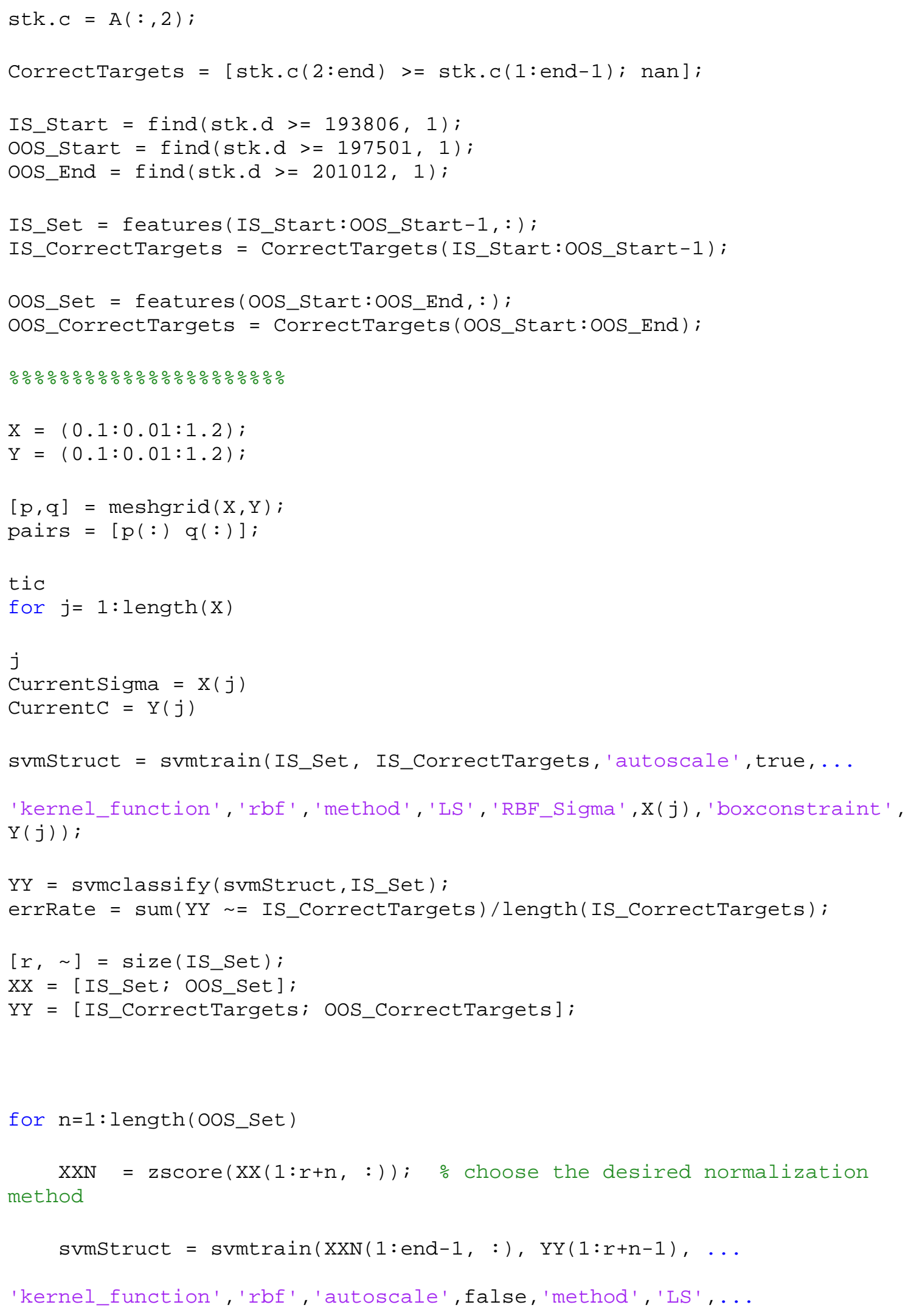


'RBF_Sigma', $X(j)$, 'boxconstraint ', $Y(j))$;

$\operatorname{Results}(n, 1)=\operatorname{svmclassify}($ svmstruct, $\operatorname{XXN}($ end, $:))$;

end

compare $=($ Results $==$ 00s_CorrectTargets $) ;$

precision $(j)=\left(\operatorname{sum}(\right.$ compare $) /$ length $($ compare $\left.){ }^{*} 100\right) ;$

CurrentPrecision $=\operatorname{precision}($ end $)$

$\operatorname{Pred}(j,:)=\operatorname{Results} ;$

end

toc

MaxPrecision $=\max ($ precision $)$

$\%$ Find the best pair

Best $=$ find $($ precision $>=\max ($ precision $))$;

Sigma $=X($ Best $)$

$C=Y($ Best $)$

figure(1)

$\%$ hold on

plot ( $X$, precision)

ylabel('Accuracy');

$\%$ hold off

\%Display Results

fprintf('Out-of-Sample Accuracy: \%6.2f \%\\n', precision); 


\section{TechnicalsequentialFeatureselection.m}

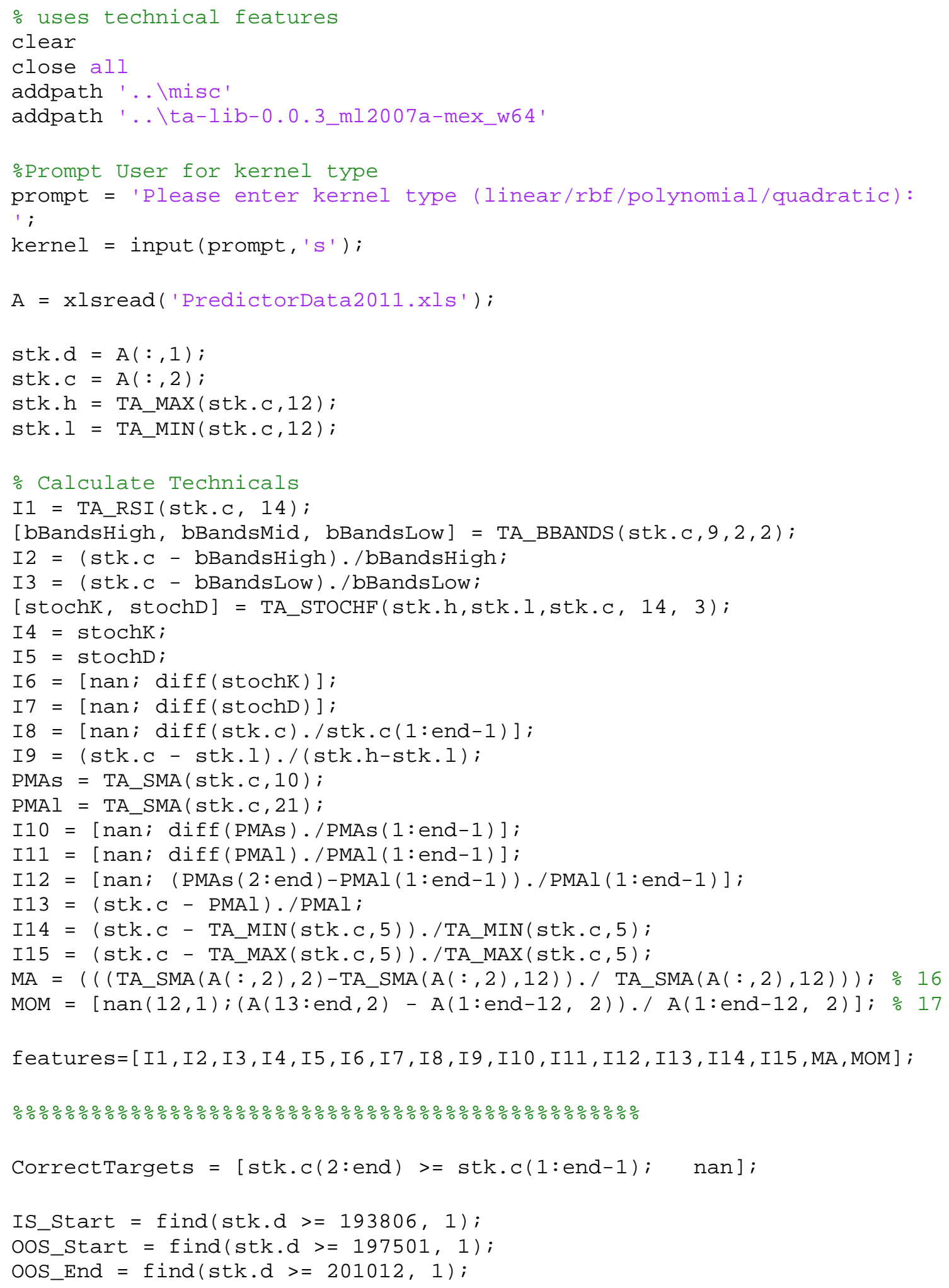




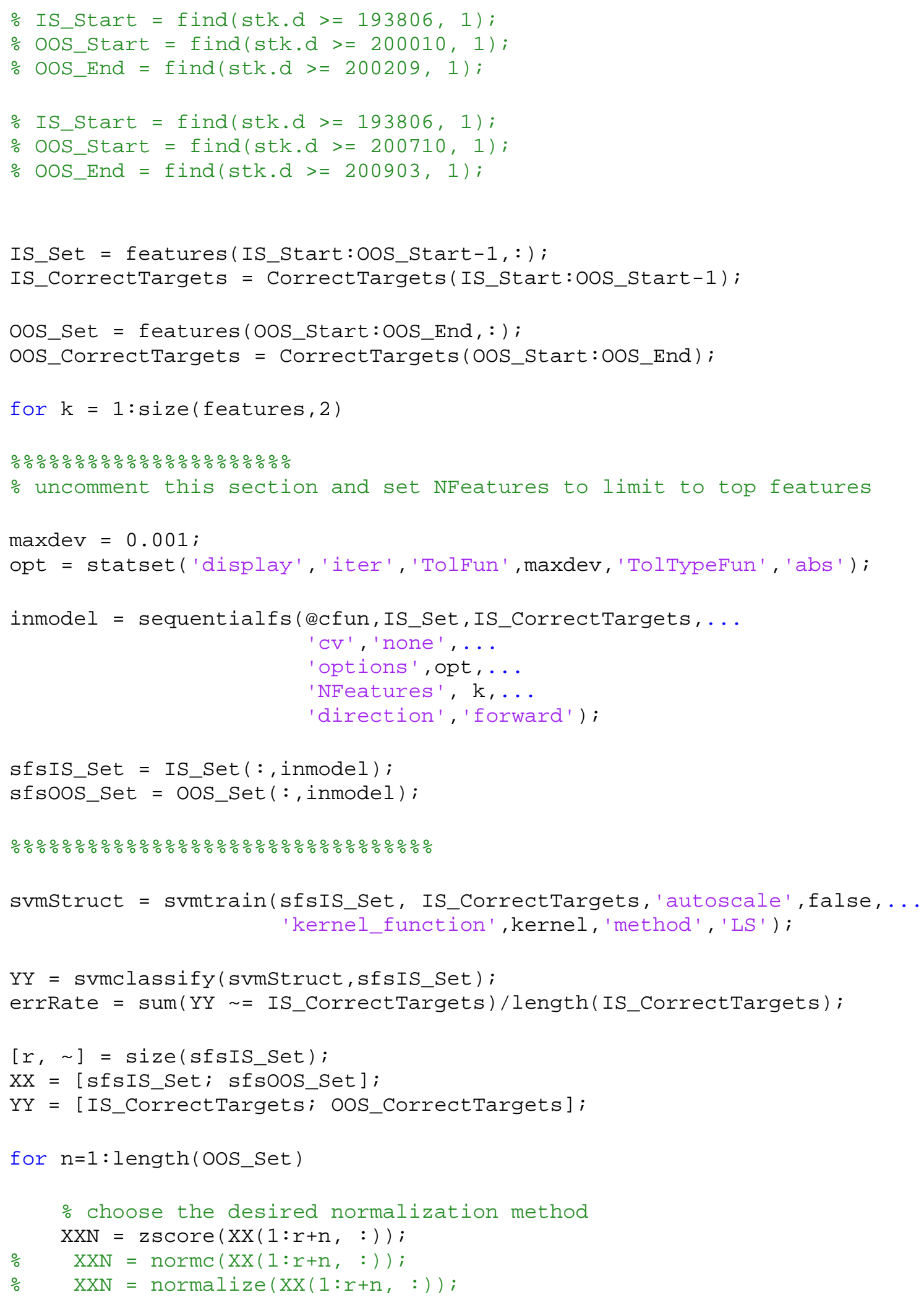




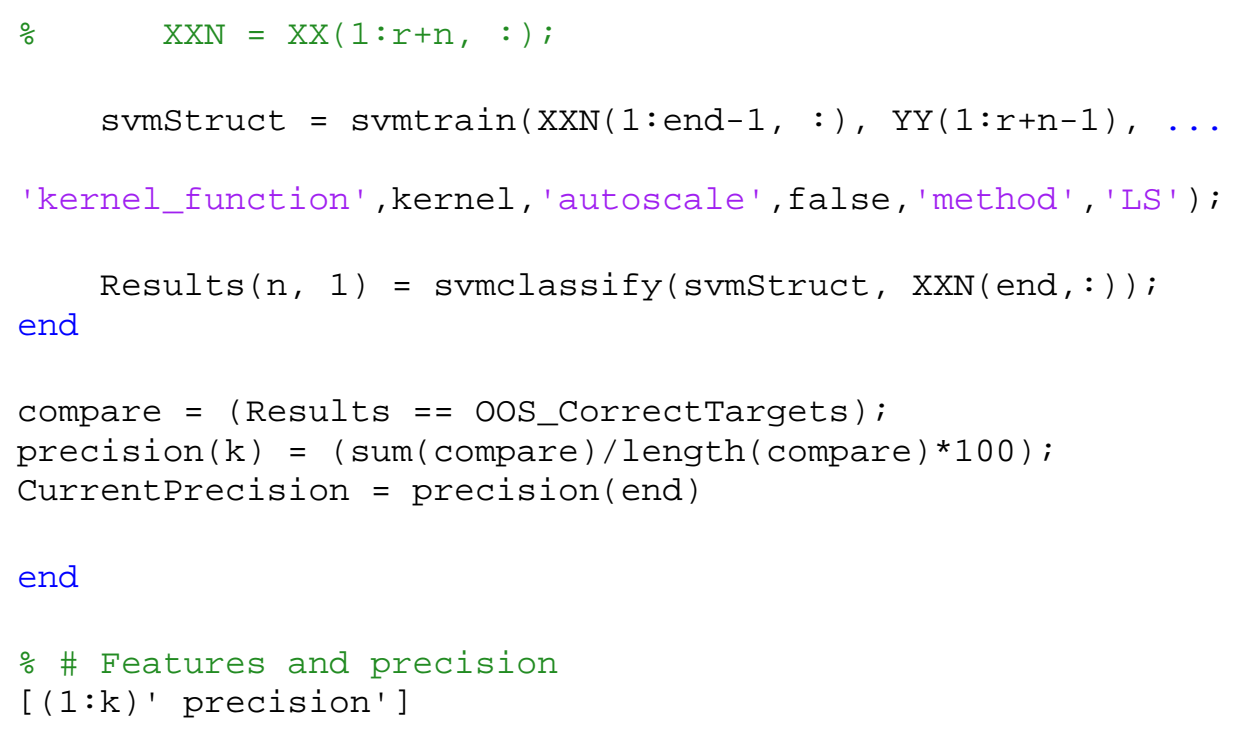




\section{TechnicalRankfeatures.m}

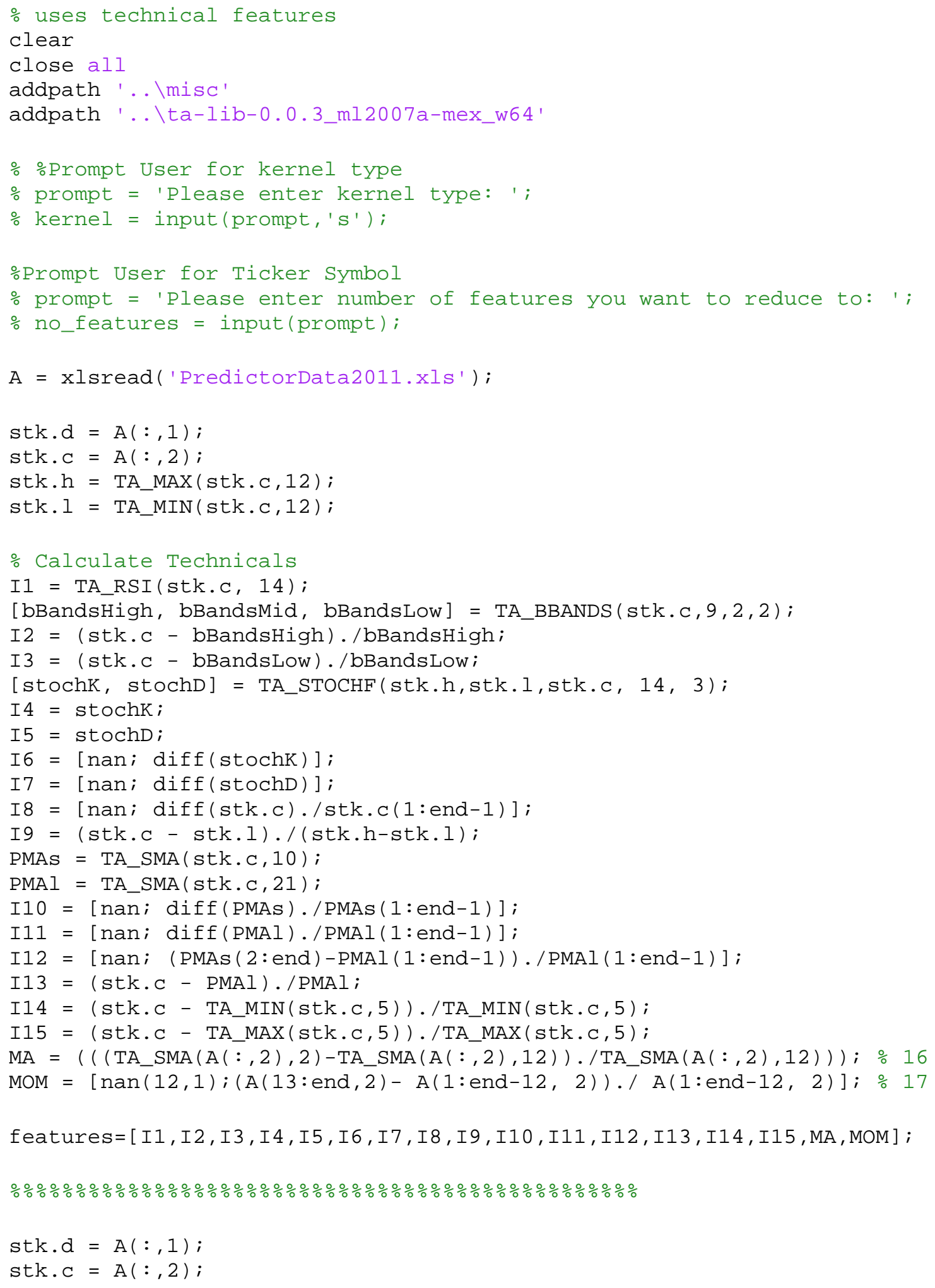




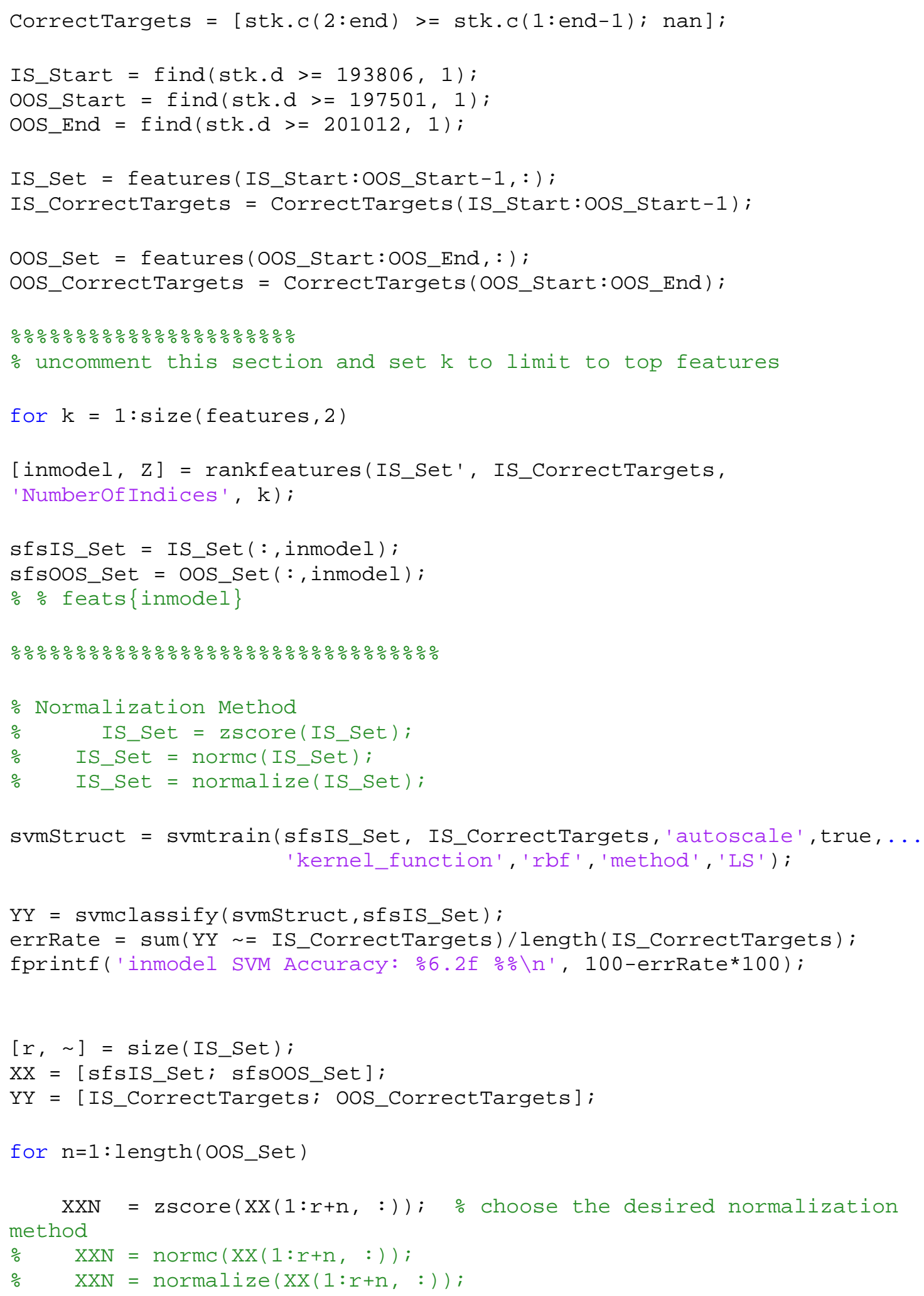




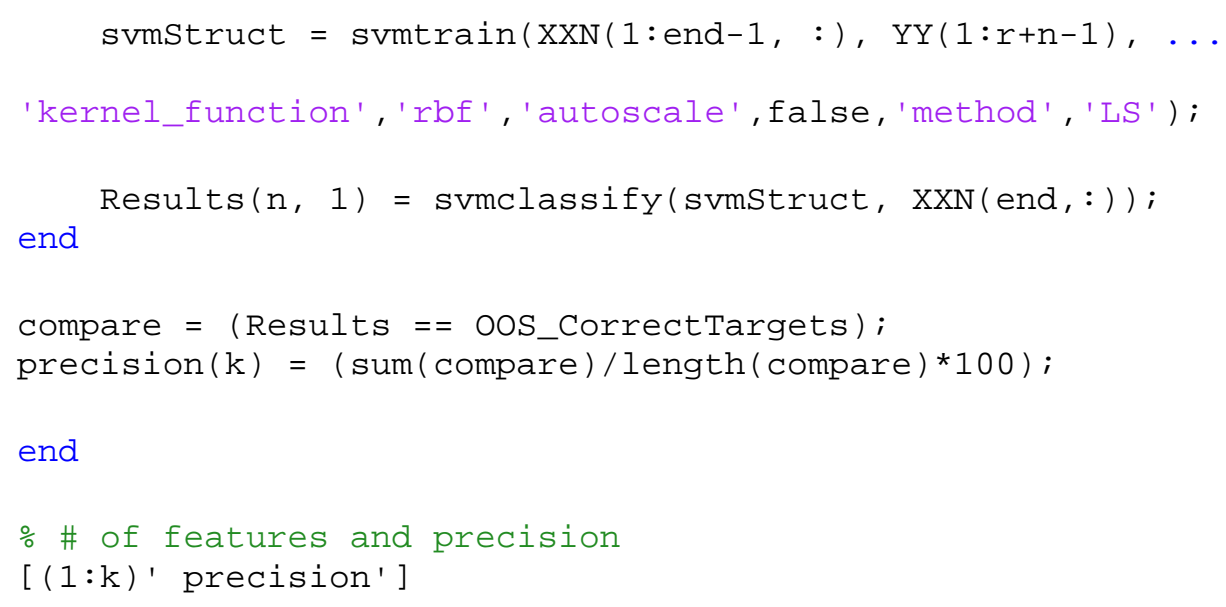




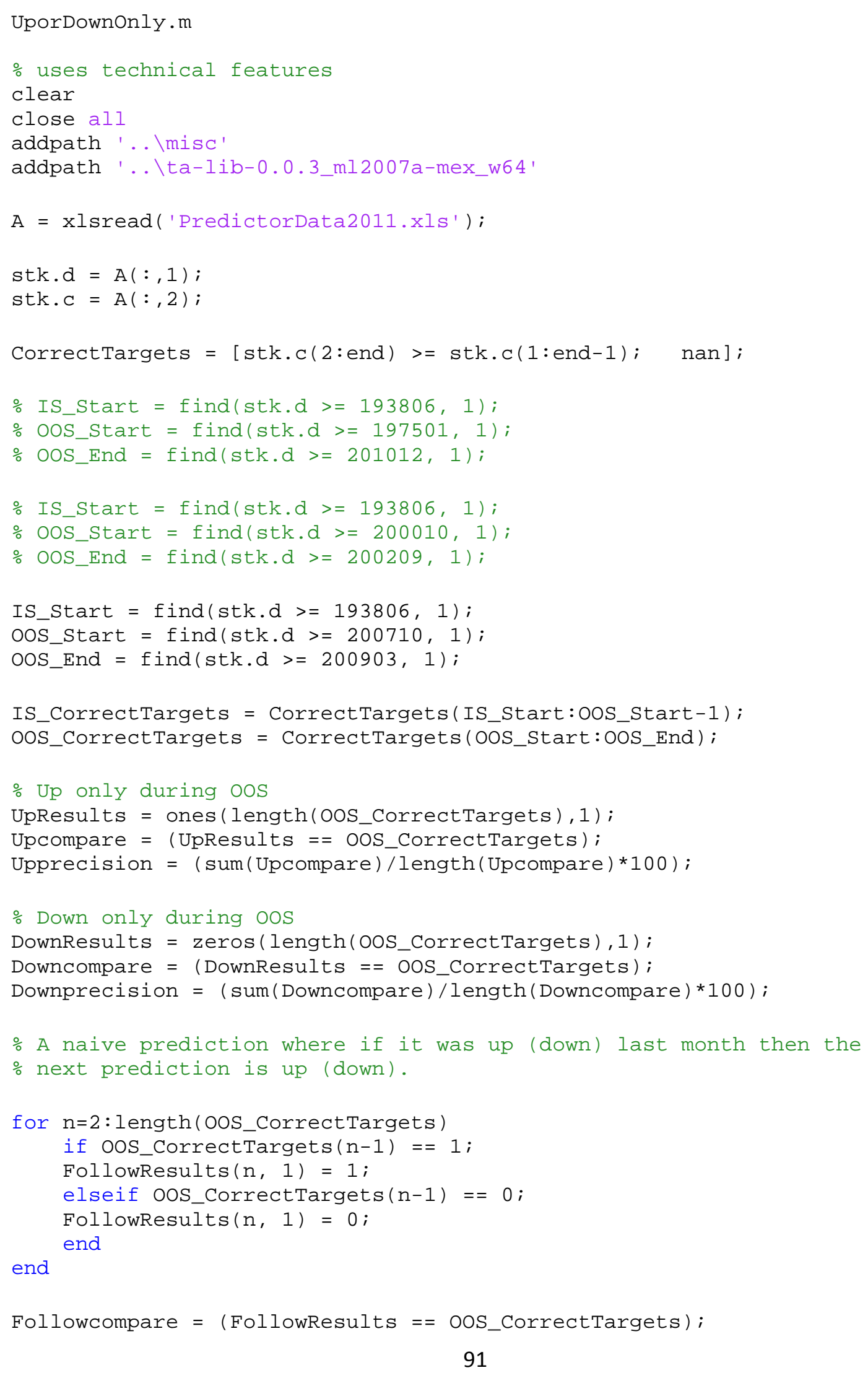




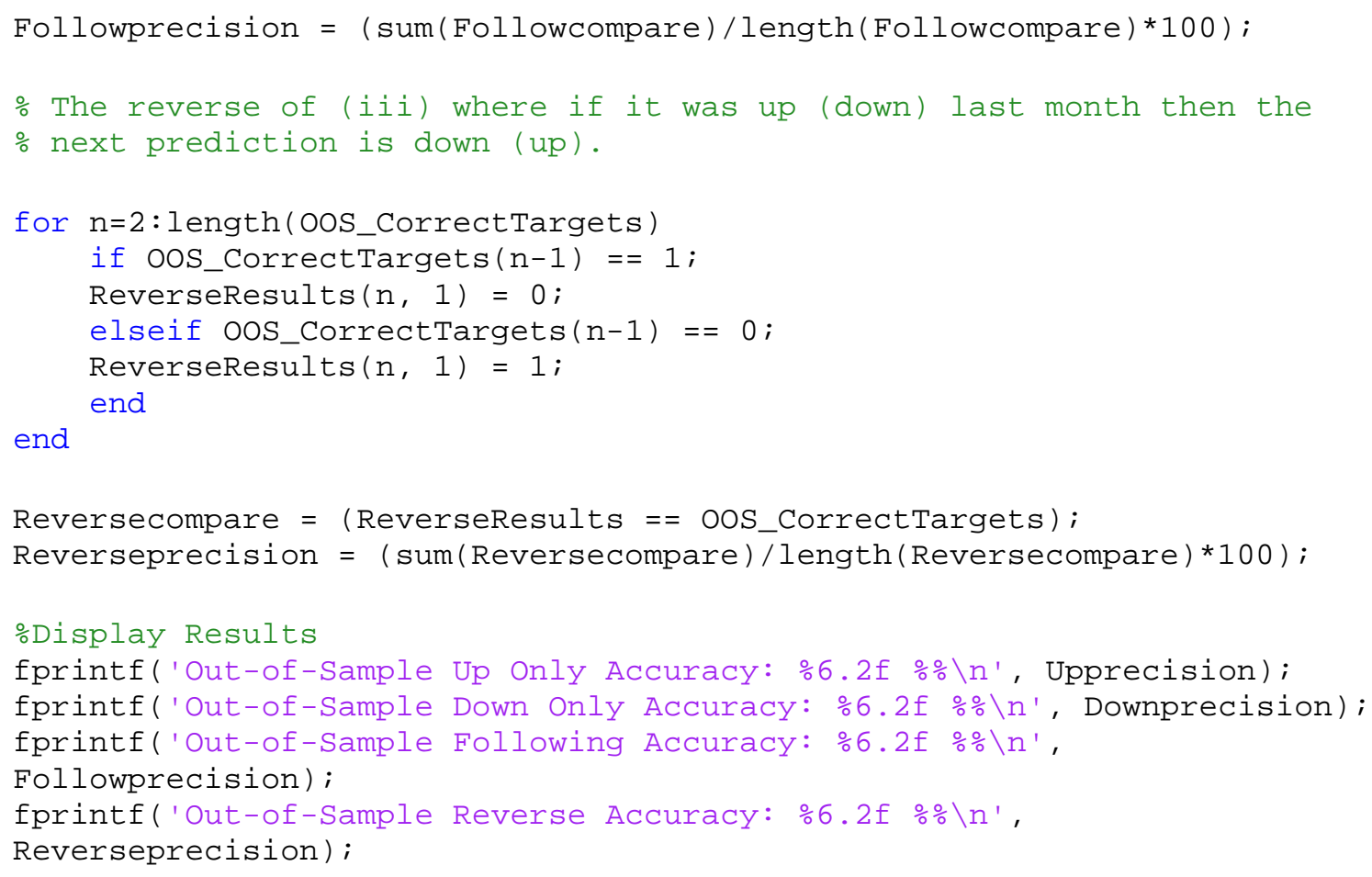




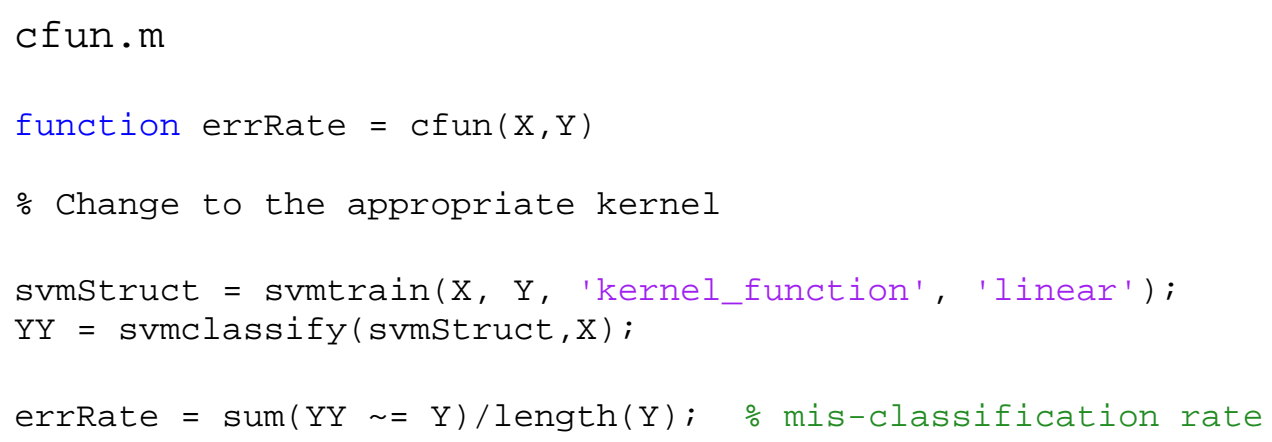


Normalize.m

function data $=$ normalize $(d)$

$\%$ the data is normalized so that max is 1 , and min is 0

data $=(d-\operatorname{repmat}(\min (d,[], 1), \operatorname{size}(d, 1), 1)) * \operatorname{spdiags}(1 . /(\max (d,[], 1)-$ $\min (d,[], 1)) ', \odot, \operatorname{size}(d, 2), \operatorname{size}(d, 2))$; 